\title{
Model-Checking Over Multi-Valued Logics
}

\author{
Marsha Chechik, Steve Easterbrook, and Victor Petrovykh \\ \{chechik, sme, victor\}ecs.toronto.edu \\ Department of Computer Science, University of Toronto \\ Toronto ON M5S 3G4, Canada
}

\begin{abstract}
Classical logic cannot be used to effectively reason about systems with uncertainty (lack of essential information) or inconsistency (contradictory information often occurring when information is gathered from multiple sources). In this paper we propose the use of quasi-boolean multi-valued logics for reasoning about such systems. We also give semantics to a multi-valued extension of CTL, describe an implementation of a symbolic multi-valued CTL model-checker

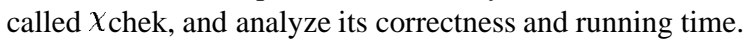

\section{Introduction}

In the last few years, model checking [10] has become established as one of the most effective automated techniques for analyzing correctness of software artifacts. Given a system and a property, a model checker builds the reachability graph (explicitly or symbolically) by exhaustively exploring the state-space of the system. Model-checking has been effectively applied to reasoning about correctness of hardware, communication protocols, software requirements and code, etc. A number of industrial model checkers have been developed, including SPIN [19], SMV [24], and Mur $\phi$ [12].

Despite their variety, existing model-checkers are typically limited to reasoning in classical logic. However, there are a number of problems in software engineering for which classical logic is insufficient. One of these is reasoning under uncertainty, or when essential information is not available. This can occur either when complete information is not known or cannot be obtained (e.g., during requirements analysis), or when this information has been removed (abstraction). Classical model-checkers typically deal with uncertainty by creating extra states, one for each value of the unknown variable and each feasible combination of values of known variables. However, this approach adds significant extra complexity to the analysis.

Classical reasoning is also insufficient for models that contain inconsistency. Inconsistency arises frequently in software engineering [15]. In requirements engineering, models are frequently inconsistent because they combine conflicting points of view. During design and implementation, inconsistency arises when integrating components developed by different people. Conventional reasoning systems cannot cope with inconsistency; the presence of a single contradiction results in trivialization - anything follows from $A \wedge \neg A$. Hence, faced with an inconsistent description and the need to perform automated reasoning, we must either discard information until consistency is achieved again, or adopt a non-classical logic. The problem with the former approach is that we may be forced to make premature decisions about which information to discard [20]. 
Although inconsistency in software engineering occurs very frequently, there have been relatively few attempts to develop automated reasoning tools for inconsistent models. Two notable exceptions are Hunter and Nuseibeh [21], who use a Quasi-Classical (QC) logic to reason about evolving specifications, and Menzies et al. [25], who use a paraconsistent form of abductive inference to reason about information from multiple points of view.

Paraconsistent logics are a promising alternative to classical reasoning — they permit some contradictions to be true, without the resulting trivialization of classical logic. The development of paraconsistent logics has been driven largely by the need for automated reasoning systems that do not give spurious answers if their databases become inconsistent. They are also of interest to mathematicians as a way of addressing the paradoxes in semantics and set theory. A number of different types of paraconsistent logic have been studied [26]. For example, relevance logics use an alternative form of entailment that requires a "relevant" connection between the antecedents and the consequents. Non-truth functional logics use a weaker form of negation so that proof rules such as disjunctive syllogism (i.e., $(A \vee B, \neg B) \vdash A)$ fail. Multi-valued logics use additional truth values to represent different types of contradiction.

Multi-valued logics provide a solution to both reasoning under uncertainty and under inconsistency. For example, we can use "no information available" and "no agreement" as logic values. In fact, model-checkers based on three-valued and four-valued logics have already been studied. For example, [8] used a three-valued logic for interpreting results of model-checking with abstract interpretation, whereas $[16,17]$ used four-valued logics for reasoning about abstractions of detailed gate or switch-level designs of circuits.

Different multi-valued logics are useful for different purposes. For example, we may wish to have several levels of uncertainty. We may wish to use different multi-valued logics to support different ways of merging information from multiple sources: keeping track of the origin of each piece of information, doing a majority vote, giving priority to one information source, etc. Thus, rather than restricting ourselves to any particular multi-valued logic, we are interested in extending the classical symbolic modelchecking procedure to enable reasoning about arbitrary multi-valued logics, as long as conjunction, disjunction and negation of the logical values are specified.

This work is part of the $\chi \mathrm{bel}^{1}$ (the Multi-Valued Belief Exploration Logics) project, outlined in [14]. The description of the system together with the description of the desired multi-valued logic and the set of correctness criteria expressed in CTL become input to our model-checker, called $\chi$ chek, which returns a value of the logic best characterizing the validity of the formula in each initial state.

The rest of this paper is organized as follows: Section 2 describes a simple thermostat system which is used as a running example throughout the paper. Section 3 gives background on CTL model-checking. Section 4 describes the types of logics that we can analyze and the ways to represent them. Section 5 describes the multi-valued transition structures and extends CTL to reasoning over them. Section 6 discusses the implementation of $\chi_{\mathrm{chek}}$, whereas Section 7 contains the analysis of its correctness and running

\footnotetext{
$\overline{1}$ pronounced "Chibel"
} 


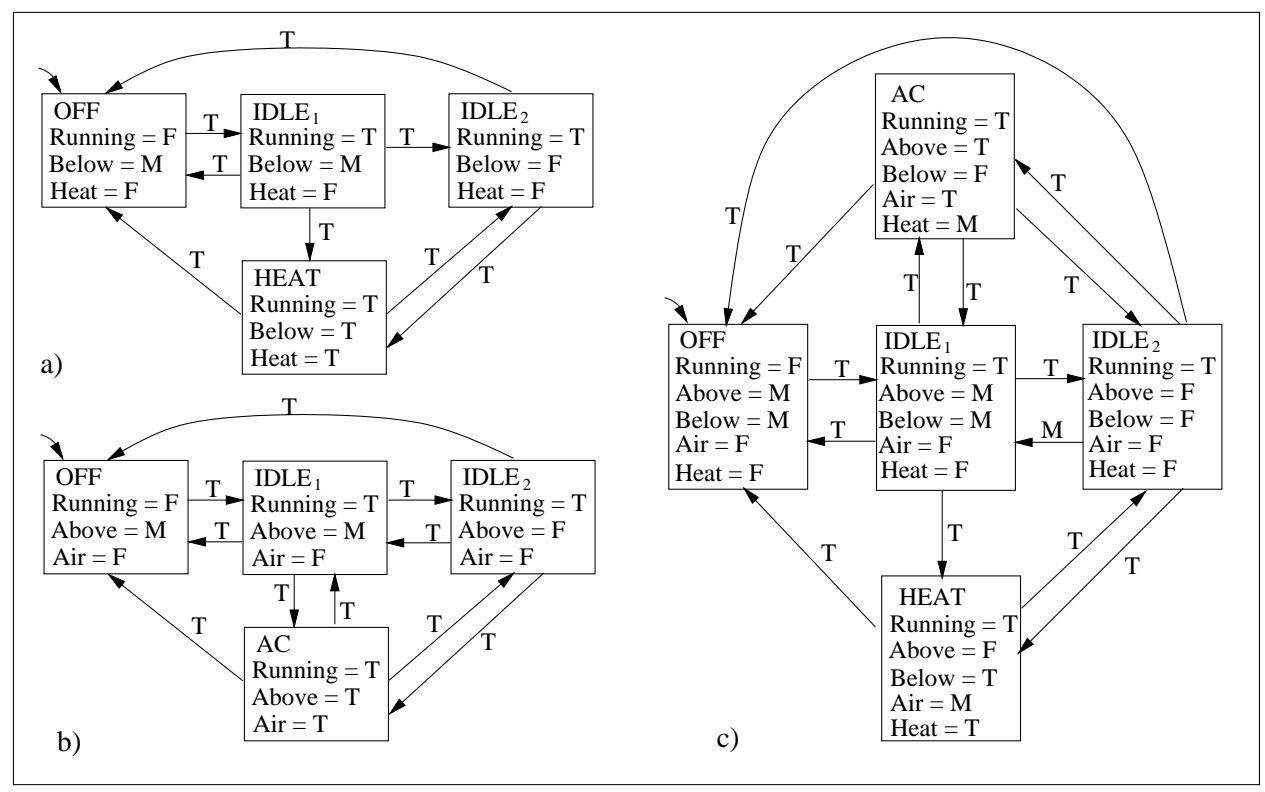

Fig. 1. Models of the thermostat. (a) Heat only; (b) AC only; (c) combined model.

time. We conclude the paper with a summary of results and outline of future work in Section 8 .

\section{Example}

Consider three models of the thermostat given in Figure 1. Figure 1(a) describes a very simple thermostat that can run a heater if the temperature falls below desired. The system has one indicator (Below), a switch to turn it off and on (Running) and a variable indicating whether the heater is running (Heat). The system starts in state $\mathrm{OFF}^{2}$ and transitions into IDLE 1 when it is turned on, where it awaits the reading of the temperature indicator. Once the temperature is determined, the system transitions either into $\operatorname{IDLE}_{2}$ or into HEAT. The value of the temperature indicator is unknown in states OFF and IDLE . $_{\text {. }}$ To model this, we could duplicate the states, assigning Below the value T in one copy and $\mathrm{F}$ in the other - the route typically taken by conventional model-checkers. Alternatively, we can model the system using the three-valued logic: T, F and M (Maybe), assigning Below the value M, as depicted in Figure 1(a) ${ }^{3}$.

We can ask this thermostat model a number of questions:

Prop. 1. Can the system transition into IDLE ${ }_{1}$ from everywhere?

Prop. 2. Can the heater be turned on when the temperature becomes below desired? Prop. 3. Can the system be turned off in every computation?

\footnotetext{
${ }^{2}$ Throughout this paper state labels are capitalized. Thus, HEAT is a state and Heat is a variable name.

${ }^{3}$ Each state in this and the other two systems in Figure 1 contains a self-loop with the value T which we omitted to avoid clutter.
} 
Figure 1(b) describes another aspect of the thermostat system - running the air conditioner. The behavior of this system is similar to that of the heater, with one difference: this system handles the failure of the temperature indicator. If the temperature reading cannot be obtained in states AC or IDLE $E_{2}$, the system transitions into state IDLE $E_{1}$.

Finally, Figure 1(c) contains a merged model, describing the behavior of the thermostat that can run both the heater and the air conditioner. In this merge, we used the same three-valued logic, for simplicity. When the individual descriptions agree that the value of a variable or transition is $\mathrm{T}(\mathrm{F})$, it is mapped into $\mathrm{T}(\mathrm{F})$ in the combined model; all other values are mapped into $\mathrm{M}$. During the merge, we used the simple invariants describing the behavior of the environment (Below $\rightarrow \neg$ Above, Above $\rightarrow \neg$ Below). Thus, the value of Below in state $\mathrm{AC}$ is inferred to be F. Note that the individual descriptions disagree on some states and transitions. For example, they disagree on a transition between $\mathrm{IDLE}_{2}$ and IDLE $E_{1}$; thus it receives the value M. Also, it is possible that the heater is on while the air conditioner is running.

Further details on the merge procedure are outside the scope of this paper, except to note that we could have chosen any of a number of different multi-valued logics to handle different combinations of values in the individual models. For example, we could have used a 9-valued logic where each value is a tuple formed from the values of the two individual models.

We can ask the combined model a number of questions that cannot be answered by either individual model, e.g.

Prop. 4. Is heat on only if air conditioning is off?

Prop. 5. Can heat be on when the temperature is above desired?

\section{CTL Model-Checking}

CTL model-checking is an automatic technique for verifying properties expressed in a propositional branching-time temporal logic called Computational Tree Logic (CTL) [10]. The system is defined by a Kripke structure, and properties are evaluated on a tree of infinite computations produced by the model of the system. The standard notation $M, s=$ $P$ indicates that a formula $P$ holds in a state $s$ of a model $M$. If a formula holds in the initial state, it is considered to hold in the model.

A Kripke structure consists of a set of states $S$, a transition relation $R \subseteq S \times S$, an initial state $s_{0} \in S$, a set of atomic propositions $A$, and a labeling function $\bar{L}: S \rightarrow \mathcal{P}(A)$. $R$ must be total, i.e, $\forall s \in S, \exists t \in S$, s.t, $(s, t) \in R$. If a state $s_{n}$ has no successors, we add a self-loop to it, so that $\left(s_{n}, s_{n}\right) \in R$. For each $s \in S$, the labeling function provides a list of atomic propositions which are True in this state.

CTL is defined as follows:

1. Every atomic proposition $a \in A$ is a CTL formula.

2. If $\varphi$ and $\psi$ are CTL formulas, then so are $\neg \varphi, \varphi \wedge \psi, \varphi \vee \psi, E X \varphi, A X \varphi, E F \varphi$, $A F \varphi, E[\varphi U \psi], A[\varphi U \psi]$.

The logic connectives $\neg, \wedge$ and $\vee$ have their usual meanings. The existential (universal) quantifier $E(A)$ is used to quantify over paths. The operator $X$ means "at the next step", 
$F$ represents "sometime in the future", and $U$ is "until". Therefore, $E X \varphi(A X \varphi)$ means that $\varphi$ holds in some (every) immediate successor of the current program state; $E F \varphi$ $(A F \varphi)$ means that $\varphi$ holds in the future along some (every) path emanating from the current state; $E[\varphi U \psi](A[\varphi U \psi])$ means that for some (every) computation path starting from the current state, $\varphi$ continuously holds until $\psi$ becomes true. Finally, we use $E G(\varphi)$ and $A G(\varphi)$ to represent the property that $\varphi$ holds at every state for some (every) path emanating from $s_{0}$. Formally,

$$
\begin{gathered}
M, s_{0} \models a \text { iff } a \in L\left(s_{0}\right) \\
M, s_{0} \models \neg \varphi \text { iff } M, s_{0} \vDash \varphi \\
M, s_{0} \models \varphi \wedge \psi \text { iff } M, s_{0} \models \varphi \wedge M, s_{0} \models \psi \\
M, s_{0} \models \varphi \vee \psi \text { iff } M, s_{0} \models \varphi \vee M, s_{0} \models \psi \\
M, s_{0} \models E X \varphi \text { iff } \exists t \in S,\left(s_{0}, t\right) \in R \wedge M, t \models \varphi \\
M, s_{0} \vDash A X \varphi \text { iff } \forall t \in S,\left(s_{0}, t\right) \in R \rightarrow M, t \models \varphi \\
M, s_{0} \models E[\varphi U \psi] \text { iff there exists some path } s_{0}, s_{1}, \ldots, \text { s.t. } \\
\exists i, i \geq 0 \wedge M, s_{i} \models \psi \\
\forall j, 0 \leq j<i \rightarrow M, s_{j} \models \varphi \\
M, s_{0} \models A[\varphi U \psi] \text { iff for every path } s_{0}, s_{1}, \ldots, \\
\exists i, i \geq 0 \wedge M, s_{i} \models \psi \wedge \\
\forall j, 0 \leq j<i \rightarrow M, s_{j} \models \varphi .
\end{gathered}
$$

where the remaining operators are defined as follows:

$$
\begin{array}{lll}
A F(\varphi) \equiv A[T U \varphi] & & \text { (def. of } A F \text { ) } \\
E F(\varphi) \equiv E[T U \varphi] & & \text { (def. of } E F) \\
A G(\varphi) \equiv \neg E F(\neg \varphi) & & \text { (def. of } A G) \\
E G(\varphi) \equiv \neg A F(\neg \varphi) & & \text { (def. of } E G \text { ) }
\end{array}
$$

Definitions of $A F$ and $E F$ indicate that we are using a "strong until", that is, $E[\varphi U \psi]$ and $A[\varphi U \psi]$ are true only if $\psi$ eventually occurs.

\section{Specifying the Logic}

Since our model checker works for different multi-valued logics, we need a way to specify the particular logic we wish to use. We can specify a logic by giving its inference rules or by defining conjunction, disjunction and negation operations on the elements of the logic. Since our goal is model-checking as opposed to theorem proving, we chose the latter approach. Further, the logic should be as close to classical as possible; in particular, the defined operations should be idempotent, commutative, etc. Such properties can be easily guaranteed if we ensure that the values of the logic form a lattice. Indeed, lattices are a natural way to specify our logics. In this section we give a brief introduction to lattice theory and describe the types of lattices used by our model-checker.

\subsection{Lattice Theory}

We introduce lattice theory here following the presentation in [2]. 


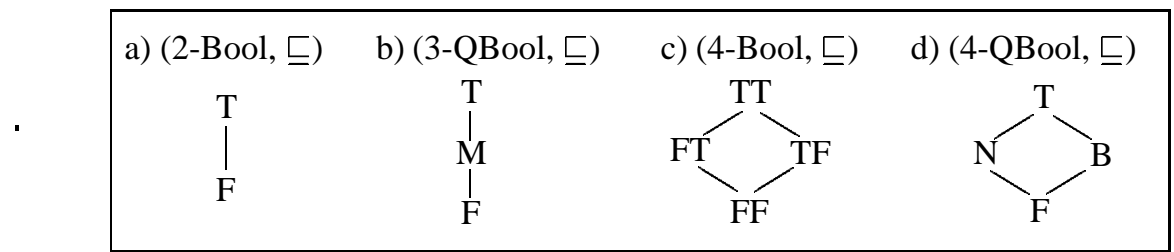

Fig. 2. Examples of logic lattices: (a) a two-valued lattice representing classical logic; (b) a threevalued lattice reflecting uncertainty; (c) a four-valued boolean lattice, a product of two (2-Bool, $\sqsubseteq$ ) lattices; (d) a four-valued quasi-boolean lattice.

\begin{tabular}{|c|c|c|c|c|}
\hline$\sqcap$ & T M F & $\sqcup$ ப $\mathrm{T}$ M & $\neg$ & \\
\hline & $\mathrm{T} \mathrm{M} \mathrm{F}$ & \begin{tabular}{l|lll}
$\mathrm{T}$ & $\mathrm{T}$ & $\mathrm{T}$ & $\mathrm{T}$ \\
\end{tabular} & $\overline{\mathrm{T}}$ & $\overline{\mathrm{F}}$ \\
\hline (a) $\mathrm{M}$ & $\mathrm{M} \mathrm{M} \mathrm{F}^{(\mathrm{b}}$ & $\mathrm{M} \mid \mathrm{T} \mathrm{M} \mathrm{M}^{(\mathrm{c})}$ & $\mathrm{M}$ & M \\
\hline $\mathrm{F}$ & $\mathrm{F} F \mathrm{~F}$ & $\mathrm{~F}$ T M F & $\mathrm{F}$ & $\mathrm{F}$ \\
\hline
\end{tabular}

Fig. 3. Tables of logic operations for (3-QBool, $\sqsubseteq)$ : (a) conjunction table; (b) disjunction table; (c) negation table.

Definition 1 Lattice is a partial order $(\mathcal{L}$, 巨) for which a unique greatest lower bound and least upper bound, denoted $a \sqcap b$ and $a \sqcup b$ exist for each pair of elements $(a, b)$.

The following are the properties of lattices:

$$
\begin{aligned}
& a \sqcup a=a \quad \text { (idempotence) } \\
& a \sqcap a=a \\
& a \sqcup b=b \sqcup a \quad \text { (commutativity) } \\
& a \sqcap b=b \sqcap a \\
& a \sqcup(b \sqcup c)=(a \sqcup b) \sqcup c \quad \text { (associativity) } \\
& a \sqcap(b \sqcap c)=(a \sqcap b) \sqcap c \\
& a \sqcup(a \sqcap b)=a \\
& a \sqcap(a \sqcup b)=a \\
& a \sqsubseteq a^{\prime} \wedge b \sqsubseteq b^{\prime} \Rightarrow a \sqcap b \sqsubseteq a^{\prime} \sqcap b^{\prime} \quad \text { (monotonicity) } \\
& a \sqsubseteq a^{\prime} \wedge b \sqsubseteq b^{\prime} \Rightarrow a \sqcup b \sqsubseteq a^{\prime} \sqcup b^{\prime}
\end{aligned}
$$

$a \sqcap b$ and $a \sqcup b$ are referred to as meet and join, representing for us conjunction and disjunction operations, respectively. Figure 2 gives examples of a few logic lattices. Conjunction and disjunction tables for the lattice in Figure 2(b) is shown in Figure 3(a)-(b). Our partial order operation $a \sqsubseteq b$ means that " $b$ is more true than $a$ ".

Definition 2 A lattice is distributive if

$$
\begin{aligned}
& a \sqcup(b \sqcap c)=(a \sqcup b) \sqcap(a \sqcup c) \quad \text { (distributivity) } \\
& a \sqcap(b \sqcup c)=(a \sqcap b) \sqcup(a \sqcap c)
\end{aligned}
$$

All lattices in Figure 2 are distributive.

Definition 3 A lattice is complete if the least upper bound and the greatest lower bound for each subset of elements of the lattice is an element of the lattice. Every complete 
lattice has a top and bottom.

$$
\begin{array}{ll}
\perp=\sqcap \mathcal{L} & (\perp \text { characterization }) \\
\top=\sqcup \mathcal{L} & (\top \text { characterization })
\end{array}
$$

In this paper we use $\mathrm{T}$ to indicate $\mathrm{T}$ of the lattice, and $\mathrm{F}$ to indicate its $\perp$, although in principle $T$ and $\perp$ might be labelled differently.

Finite lattices are complete by definition. Thus, all lattices representing finite-valued logics are complete.

Definition 4 A complete distributive lattice is called a complete Boolean lattice if every element $a \in \mathcal{L}$ has a unique complement $\neg a \in \mathcal{L}$ satisfying the following conditions:

$$
\begin{aligned}
& \neg \neg a=a \quad \text { ( } \neg \text { involution) } \quad a \sqsubseteq b \Leftrightarrow \neg a \sqsupseteq \neg b \quad(\neg \text { antimonotonic) } \\
& \neg(a \sqcap b)=\neg a \sqcup \neg b \quad \text { (de Morgan) } \quad a \sqcap \neg a=\perp \quad \text { ( } \begin{array}{l}
\text { contradiction) } \\
\text { ) }
\end{array} \\
& \neg(a \sqcup b)=\neg a \sqcap \neg b \quad \text { (de Morgan) } \quad a \sqcup \neg a=\top \quad \text { ( }
\end{aligned}
$$

In fact, $\neg$ involution, de Morgan and antimonotonic laws follow from $\neg$ contradiction and $\neg$ exhaustiveness.

Definition 5 A product of two lattices $\left(\mathcal{L}_{1}, \sqsubseteq\right),\left(\mathcal{L}_{2}, \sqsubseteq\right)$ is a lattice $\left(\mathcal{L}_{1} \times \mathcal{L}_{2}\right)$, with the ordering $\sqsubseteq$ holding between two pairs iff it holds for each component separately, i.e.

$$
(a, b) \sqsubseteq\left(a^{\prime}, b^{\prime}\right) \Leftrightarrow a \sqsubseteq a^{\prime} \wedge b \sqsubseteq b^{\prime} \quad \text { ( } \text { of pairs) }
$$

Bottom, top, complement, meet and join in the product lattice are component-wise extensions of the corresponding operations of the component lattices. Product of two lattices preserves their distributivity, completeness and boolean properties. For example, out of the four lattices in Figure 2, only (2-Bool, $\sqsubseteq)$ and (4-Bool, $\sqsubseteq)$ are boolean. The former is boolean because $\neg \mathrm{T}=\mathrm{F}, \neg \mathrm{F}=\mathrm{T}$. The latter is a product of two (2-Bool, $\sqsubseteq$ ) lattices and thus is complete, distributive and boolean. The lattice (3-QBool, $\sqsubseteq$ ) is not boolean because $\neg \mathrm{M}=\mathrm{M}$, and $\mathrm{M} \sqcap \neg \mathrm{M} \neq \perp$.

\subsection{Quasi-Boolean Lattices}

Definition 6 A distributive lattice ( $\mathcal{L}$, $\sqsubseteq$ ) is quasi-boolean [4] (also called de Morgan [13]) if there exists a unary operator $\neg$ defined for it, with the following properties ( $a, b$ are elements of $(\mathcal{L}, \sqsubseteq))$ :

$$
\begin{aligned}
& \neg(a \sqcap b)=\neg a \sqcup \neg b \quad \text { (de Morgan) } \quad \neg \neg a=a \quad \text { ( } \rightarrow \text { involution) } \\
& \neg(a \sqcup b)=\neg a \sqcap \neg b \quad a \sqsubseteq b \Leftrightarrow \neg a \sqsupseteq \neg b \quad \text { ( } \neg \text { antimonotonic) }
\end{aligned}
$$

Thus, $\neg$ a is a quasi-complement of $a$.

Therefore, all boolean lattices are also quasi-boolean, whereas the converse is not true. Logics represented by quasi-boolean lattices will be referred to as quasi-boolean logics.

Theorem 1 A product of two quasi-boolean lattices is quasi-boolean. 
Proof:

Refer to the Appendix for proof of this and other theorems of this paper.

For example, the lattice (3-QBool, ఏ), first defined in [23], and all its products are quasi-boolean. We refer to $n$-valued boolean lattices as ( $n$-Bool, $\sqsubseteq)$ and to $n$-valued quasi-boolean lattices as ( $n$-QBool, $\sqsubseteq)$. (4-QBool, $\sqsubseteq)$ is a lattice for a logic proposed by Belnap for reasoning about inconsistent databases $[3,1]$. This lattice is quasi-boolean $(\neg \mathrm{N}=\mathrm{N} ; \neg \mathrm{B}=\mathrm{B})$ and thus not isomorphic to (4-Bool, $\sqsubseteq$ ).

In the rest of this paper we assume that the negation operator given for our logic makes the lattice quasi-boolean. Figure 3(c) gives the negation function for lattice (3QBool, Ђ).

What do quasi-boolean lattices look like? Below we define lattices which are (geometrically) horizontally-symmetric and show that, with negation defined by the horizontal symmetry, this is a sufficient condition for quasi-booleanness. We define:

Definition 7 A lattice $(\mathcal{L}$, $\sqsubseteq)$ is horizontally-symmetric if there exists a bijective function $H$ such that for every pair $a, b \in \mathcal{L}$,

$$
\begin{aligned}
a \sqsubseteq b \Leftrightarrow & H(a) \sqsupseteq H(b) & & \text { (order - embedding) } \\
& H(H(\bar{a}))=a & & \text { (H involution) }
\end{aligned}
$$

Theorem 2 Let $(\mathcal{L}, \sqsubseteq)$ be a horizontally-symmetric lattice. Then the following hold for any two elements $a, b \in \mathcal{L}$ :

$$
\begin{aligned}
& H(a \sqcap b)=H(a) \sqcup H(b) \\
& H(a \sqcup b)=H(a) \sqcap H(b)
\end{aligned}
$$

Thus, horizontal symmetry is a sufficient condition for the corresponding lattice to be quasi-boolean, with $\neg a=H(a)$ for each element of the lattice, since it guarantees antimonotonicity and involution by definition, and de Morgan laws via Theorem 2.

\section{Multi-Valued CTL Model-Checking}

In this section we extend the notion of boolean model-checking described in Section 3 by defining multi-valued Kripke structures and multi-valued CTL.

\subsection{Defining the Model}

A state machine $M$ is a multi-valued Kripke ( $\chi$ Kripke) structure if $M=\left(S, S_{0}, R, I, A, L\right)$, where

- $L$ is a quasi-boolean logic represented by a lattice $(\mathcal{L}, \sqsubseteq)$.

- $A$ is a (finite) set of atomic propositions, otherwise referred to as variables (e.g. Running, Below, Heat in Figure 1(a)). For simplicity, we assume that all variables are of the same type, ranging over the values of the logic.

- $S$ is a (finite) set of states. States are not explicitly labeled - each state is uniquely identified by its variable/value mapping. Thus, two states cannot have the same mapping. However, we sometimes use state labels as a shorthand for the respective vector of values, as we did in the thermostat example. 


$$
\begin{aligned}
\neg A X \varphi & =E X(\neg \varphi) & & \text { (negation of "next") } \\
A[\perp U \varphi] & =E[\perp U \varphi]=\varphi & & \text { ( } \perp \text { "until") } \\
A[\varphi U \psi] & =\psi \vee(\varphi \wedge A X A[\varphi U \psi] \wedge E X A[\varphi U \psi]) & & \text { (AU fixpoint) } \\
E[\varphi U \psi] & =\psi \vee(\varphi \wedge E X E[\varphi U \psi]) & & \text { (EU fixpoint) }
\end{aligned}
$$

Fig. 4. Properties of CTL operators (from [22, 6]).

- $S_{0} \subseteq S$ is the non-empty set of initial states.

- Each transition $(s, t)$ in $M$ has a logical value in $\mathcal{L}$. Thus, $R: S \times S \rightarrow \mathcal{L}$ is a total function assigning a truth value from the logic $L$ to each possible transition between states. The value of $(s, t)$ in $M$ is thus referred to as $R^{M}(s, t)$, or, when $M$ is clear from the context, simply as $R(s, t)$. Note that a $\chi$ Kripke structure is a completely connected graph. We also ensure that there is at least one non-false transition out of each state, extending the classical notion of Kripke structures. Formally,

$$
\forall s \in S, \exists t \in S \text {, s.t. } R(s, t) \neq \perp
$$

To avoid clutter, we follow the convention of finite-state machines of not drawing $\mathrm{F}$ transitions. Thus, in Figure 1(a), transition between IDLE ${ }_{2}$ and IDLE Is $_{1}$ F, whereas in Figure 1(c) this transition is $\mathrm{M}$.

- $I: S \times A \rightarrow \mathcal{L}$ is a total function that maps a state $s$ and an atomic proposition (variable) $a$ to a truth value $v$ of the logic. For a given variable $a$, we will write $I$ as $I_{a}: S \rightarrow \mathcal{L}$. Central to the rest of the paper will be a notion of partitions of the state space w.r.t. a variable $a$, referred to as $I_{a}^{-1}: \mathcal{L} \rightarrow 2^{S}$. A partition has the following properties:

$$
\begin{array}{ll}
\forall a \in A, \forall v_{1}, v_{2} \in \mathcal{L}: v_{1} \neq v_{2} \Rightarrow\left(I_{a}^{-1}\left(v_{1}\right) \cap I_{a}^{-1}\left(v_{2}\right)=\emptyset\right) & \text { (disjointness) } \\
\forall a \in A, \forall s \in S, \exists v \in \mathcal{L}: s \in I_{a}^{-1}(v) & \text { (cover) }
\end{array}
$$

Finally, we refer to a value that a variable $a$ takes in state $s$ as $s \llbracket a \rrbracket^{M}$, or, when $M$ is clear from context, simply as $s \llbracket a \rrbracket$.

\subsection{Multi-Valued CTL}

Here we give semantics of CTL operators on a $\chi$ Kripke structure $M$ over a quasi-boolean logic $L$. We will refer to this language as multi-valued $C T L$, or $\chi C T L . L$ is described by a finite, quasi-boolean lattice $(\mathcal{L}, \sqsubseteq)$, and thus the conjunction $\wedge$ ( $\sqcap$ operation of the lattice), disjunction $\vee(\sqcup$ operation of the lattice) and negation $\neg$ operations are available. We also define the material implication $\rightarrow$ as follows:

$$
a \rightarrow b \equiv \neg a \vee b \quad \text { (definition of } \rightarrow \text { ) }
$$

In extending the CTL operators, we want to ensure that the expected CTL properties, given in Figure 4, are still preserved. Note that the $A U$ fixpoint is somewhat unusual because it includes an additional conjunct, $E X A[f U g]$. The reason for this term is to preserve a "strong until" semantics for states that have no outgoing T transitions. This term was introduced by [6] for reasoning about non-Kripke structures. 


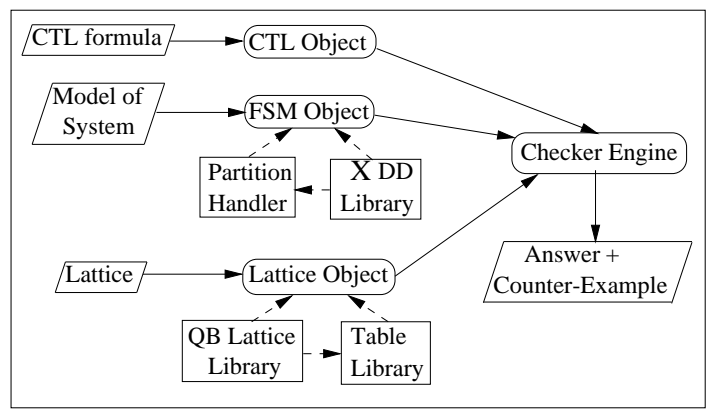

Fig. 5. Architecture of $\chi_{\text {chek. }}$

We start defining $\chi$ CTL by giving the semantics of propositional operators. Here, $s$ is a state and $v \in \mathcal{L}$ is a logic value:

$$
\begin{aligned}
s \llbracket a \rrbracket & =I_{a}(s) \\
s \llbracket \neg \varphi \rrbracket & =\neg s \llbracket \varphi \rrbracket \\
s \llbracket \varphi \wedge \psi \rrbracket & =s \llbracket \varphi \rrbracket \wedge s \llbracket \psi \rrbracket \\
s \llbracket \varphi \vee \psi \rrbracket & =s \llbracket \varphi \rrbracket \vee s \llbracket \psi \rrbracket
\end{aligned}
$$

We proceed by defining $E X$ and $A X$ operators. Recall from Section 3 that these operators were defined using existential and universal quantification over next states. We extend the notion of quantification for multi-valued reasoning by using conjunction and disjunction operators. This treatment of quantification is standard [3, 27]. The semantics of $E X$ and $A X$ operators is given below:

$$
\begin{aligned}
& s \llbracket E X \varphi \rrbracket=\bigvee_{t \in S}(R(s, t) \wedge t \llbracket \varphi \rrbracket) \\
& s \llbracket A X \varphi \rrbracket=\bigwedge_{t \in S}(R(s, t) \rightarrow t \llbracket \varphi \rrbracket)
\end{aligned}
$$

Theorem 3 Definitions of $s \llbracket E X \varphi \rrbracket$ and $s \llbracket A X \varphi \rrbracket$ preserve the negation of "next" property, i.e.

$$
\forall s \in S, \neg s \llbracket A X \varphi \rrbracket=s \llbracket E X \neg \varphi \rrbracket
$$

Finally, we define $A U$ and $E U$ operators using the $A U$ and $E U$ fixpoint properties:

$$
\begin{aligned}
& s \llbracket E[\varphi U \psi] \rrbracket=s \llbracket \psi \rrbracket \vee(s \llbracket \varphi \rrbracket \wedge s \llbracket E X E[\varphi U \psi] \rrbracket) \\
& s \llbracket A[\varphi U \psi] \rrbracket=s \llbracket \psi \rrbracket \vee(s \llbracket \varphi \rrbracket \wedge s \llbracket A X A[\varphi U \psi] \rrbracket \wedge s \llbracket E X A[\varphi U \psi] \rrbracket)
\end{aligned}
$$

The remaining CTL operators, $A F(\varphi), E F(\varphi), A G(\varphi), E G(\varphi)$ are the abbreviations for $A[\top U \varphi], E[\top U \varphi], \neg E F(\neg \varphi), \neg A F(\neg \varphi)$, respectively.

\section{6 $\chi$ chek: A Multi-Valued Model-Checker}

In this section we describe our implementation of a multi-valued CTL model-checker.

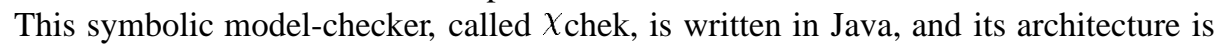
depicted in Figure 5. The checking engine receives the $\chi \mathrm{CTL}$ formulas to verify, the model of the system represented as an $\chi$ Kripke structure, and a lattice of logic values, 
and checks whether the specified property holds, returning an answer (one of the values of the passed lattice) and a counter-example, if appropriate. $\chi$ chek uses four supplementary libraries: $\chi$ DDs (a multi-valued extension of binary decision diagrams [5], described in [9]), a library for handling quasi-boolean lattices, a partition handler and a table inverter. The functionality of the latter two libraries is described below.

\subsection{Table Library}

The Table library contains several tables, indexed by the elements of the lattice, that give quick access to a variety of operations on lattice elements. In order to enable this indexing, we define Ord $: \mathcal{L} \rightarrow \mathbb{N}-$ a total order on the elements of our lattice $(\mathcal{L}, \sqsubseteq)$. Ord is a bijection, mapping each element $v \in \mathcal{L}$ onto the set $\{1 \ldots|\mathcal{L}|\}$. For example, we can order the elements of the lattice (3-QBool, $\sqsubseteq)$ as follows:

$$
\operatorname{Ord}(\mathrm{T})=1 \quad \operatorname{Ord}(\mathrm{M})=2 \quad \operatorname{Ord}(\mathrm{F})=3
$$

This ordering is referred to as $\mathrm{T}<\mathrm{M}<\mathrm{F}$.

Using Ord and the primitive lattice operations, we compute inverted tables: given a value, these tables give pairs of elements yielding this value when the corresponding operation is performed on them. Three inverted tables, InvTable $e_{\wedge}$, InvTable $_{\rightarrow}$ and InvTable $\vee$ are computed, one for each operator. For a table $T$ and a value $v$, we use notation $T_{v}$ to indicate an element associated with value $v$. InvTable $e_{v}$ is defined as

$$
\forall v \in \mathcal{L}, \text { InvTable } \mathrm{e}_{v}=\left\{\left(v_{1}, v_{2}\right) \mid v_{1}, v_{2} \in \mathcal{L} \vee v_{1} \wedge v_{2}=v\right\}
$$

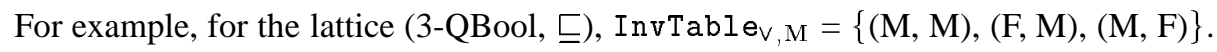
InvTable $e_{\wedge}$ and InvTable $\rightarrow$ are defined similarly.

Afterwards, we build generalized versions of the inverted tables for conjunction and disjunction over more than two operands. We call them BigOPTable $e_{\wedge}$ and BigOPTable ${ }_{\vee}$ Given a logic value $v$, BigOPTable $e_{o p, v}$ gives sets of logic values, where the corresponding operation $o p$ over the elements of the set yields $v$. For example, for the lattice (3QBool, Б), BigOPTable $e_{\vee}$ is $\{\{\mathrm{T}\},\{\mathrm{T}, \mathrm{M}\},\{\mathrm{T}, \mathrm{F}\},\{\mathrm{T}, \mathrm{M}, \mathrm{F}\}\} . \mathrm{BigOPTable}_{\vee}$ is defined as

$$
\forall v \in \mathcal{L}, \text { BigOPTable }_{\vee, v}=\{V \mid V \in \mathcal{P}(\mathcal{L}) \wedge \bigvee V=v\}
$$

BigOPTable $e_{\wedge}$ is defined similarly. Since the generalized tables will be used only for computing commutative operations, we will not need to define $\mathrm{BigOPTable}_{\rightarrow}$.

\subsection{The Partition Handler}

Central to the design of $\chi$ chek is the notion of partition and cover. A cover (satisfying the cover property given in Section 5.1) separates the states of the model into subsets corresponding to the different values of the logic for a proposition $\varphi$. If sets of states in a cover are mutually disjoint, we call it a partition. Disjointness property is also given in Section 5.1).

More formally, a cover $\llbracket \varphi \rrbracket^{M}$ for a property $\varphi$ and a machine $M$ is a tuple of sets such that the $i$ th element of the tuple is a set of states where $\varphi$ has the value $\operatorname{Ord}^{-1}(i)$ 
in $M$. When the choice of $M$ is clear, we omit it, referring to the above as $\llbracket \varphi \rrbracket$. For a value $v \in \mathcal{L}$, we write $\llbracket \varphi \rrbracket v$ to indicate the set of states associated with $v$. Note that if $\varphi$ is an arbitrary $\chi C T L$ property, then $\llbracket \varphi \rrbracket$ is a partition. For an example in Figure 1(a) and ordering $\mathrm{T}<\mathrm{M}<\mathrm{F}, \llbracket$ Below $\rrbracket=\left(\{\mathrm{HEAT}\},\left\{\mathrm{OFF}, \mathrm{IDLE}_{1}\right\},\left\{\mathrm{IDLE}_{2}\right\}\right)$.

Further, we define a predecessor function pred which receives a cover $\llbracket \varphi \rrbracket$ and an operator $o p \in\{\wedge, \rightarrow\}$ and returns a cover: a state $s$ is associated with value $v_{1}$ in $\operatorname{pred}(\llbracket \varphi \rrbracket, o p)$ iff $s$ has a successor state $t$ where $\varphi$ has value $v_{2}$, and $R(s, t)$ op $v_{2}=v_{1}$. The function is given in Figure 6. For the lattice (3-QBool, $\sqsubseteq$ ), its ordering $\mathrm{T}<\mathrm{M}<\mathrm{F}$ and the model in

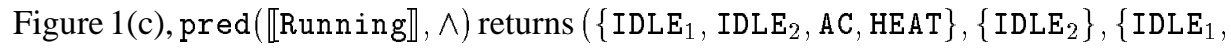
$\left.\left.\mathrm{IDLE}_{2}, \mathrm{AC}, \mathrm{HEAT}, \mathrm{OFF}\right\}\right)$.

We further define functions doOp and doBig0p, described in Figure 6. These functions evaluate the expression using the appropriate table (InvTable $e_{o p}$ or BigOPTable $e_{o p}$ ). Given covers $\llbracket \varphi \rrbracket$ and $\llbracket \psi \rrbracket$, doOP returns a cover for $\varphi$ op $\psi$. If $\llbracket \varphi \rrbracket$ and $\llbracket \psi \rrbracket$ are partitions, then so is $\varphi$ op $\psi$. For the lattice (3-QBool, $\sqsubseteq$ ) and the model in Figure 1(c), $\llbracket$ doOP $(\llbracket$ Above $\rrbracket, \vee, \llbracket B e l o w \rrbracket) \rrbracket T$ returns a set of states in which Above $\vee$ Below is $T$, namely, $\{\mathrm{AC}, \mathrm{HEAT}\}$.

doBigOP $(o p, \llbracket \varphi \rrbracket)$ computes a conjunction or a disjunction over a set of states. Recall that BigOPTable $e_{o p, v}$ computes sets of logic values such that the operation $o p$ performed on them yields $v$. An operation $o p \varphi$ over a set of states should yield $v$ if the value of $\varphi$ is in BigOPTable $e_{o p, v}$ in each state in the set. Thus, for each $V$ in BigOPTable $e_{o p, v}$, we compute the intersection of states for which $\varphi$ has a value in $V$ and subtract the union of states in which $\varphi$ does not have a value in $V$. 【result $\rrbracket v$ is the union of all states computed via the above process for all $V$ in BigOPTable $e_{o p, v}$. For the model in Figure 1(c), \doBigOP $(\vee, \llbracket$ Heat $\rrbracket) \rrbracket T$ returns $\{$ HEAT $\}$. Note that if $\llbracket \varphi \rrbracket$ is a partition, $\operatorname{doBigOP}(o p, \llbracket \varphi \rrbracket)$ simply returns a partition $\llbracket \psi \rrbracket$, where $\llbracket \psi \rrbracket v=\llbracket \varphi \rrbracket v$ for each $v \in \mathcal{L}$.

\subsection{Algorithm for $\chi_{\text {chek }}$}

The high-level algorithm, inspired by Bultan's symbolic model checker for infinite-state systems $[6,7]$ and an abstract model-checker of [8], is given in procedure Check in Figure 6.

The algorithm recursively goes through the structure of the property under the analysis, associating each subproperty $\varphi$ with a partition $\llbracket \varphi \rrbracket$. In fact, Check always returns partitions on the state-space (see Theorem 5). For the example in Figure 1(c) and the lattice ordering $\mathrm{T}<\mathrm{M}<\mathrm{F}$,

$$
\begin{array}{ll}
\text { Check }(\neg \text { Running }) & =\left(\{\text { OFF }\},\{\},\left\{\operatorname{IDLE}_{1}, \operatorname{IDLE}_{2}, \mathrm{AC}, \mathrm{HEAT}\right\}\right) \\
\operatorname{Check}(\text { Above } \vee \text { Below }) & =\left(\left\{\mathrm{AC}_{\mathrm{HEAT}}\right\},\left\{\mathrm{OFF}_{1} \mathrm{IDLE}_{1}\right\},\left\{\mathrm{IDLE}_{2}\right\}\right) \\
\operatorname{Check}(A X \neg \text { Heat }) & =\left(\{\mathrm{OFF}, \mathrm{AC}\},\{\},\left\{\mathrm{IDLE}_{1}, \mathrm{IDLE}_{2}, \mathrm{HEAT}\right\}\right)
\end{array}
$$

Function QUntil determines the value of $E[\varphi U \psi]$ and $A[\varphi U \psi]$ using a fixpoint algorithm given in Figure 6. The lowest ("most false") value that $A[\varphi U \psi] / E[\varphi U \psi]$ can have in each state $s$ is $s \llbracket \psi \rrbracket$. Thus, $Q U_{0}$ is equal to $\llbracket \psi \rrbracket$. At each iteration, the algorithm computes $\mathrm{EXTerm}_{i+1}$, equal to $E X Q U_{i}$. If the function is called with the universal quantifier, then it also computes $\mathrm{AXTerm}_{i+1}$, equal to $A X Q U_{i}$. Otherwise, $\mathrm{AXTerm}_{i+1}$ is not necessary, and thus we let $\mathrm{AXTerm}_{i+1}$ be $\llbracket \varphi \rrbracket$. $A X Q U_{i}$ and $E X Q U_{i}$ are computed by invoking the function $\mathrm{D} O \mathrm{BigOP}$ and passing it the result of the appropriate pred 


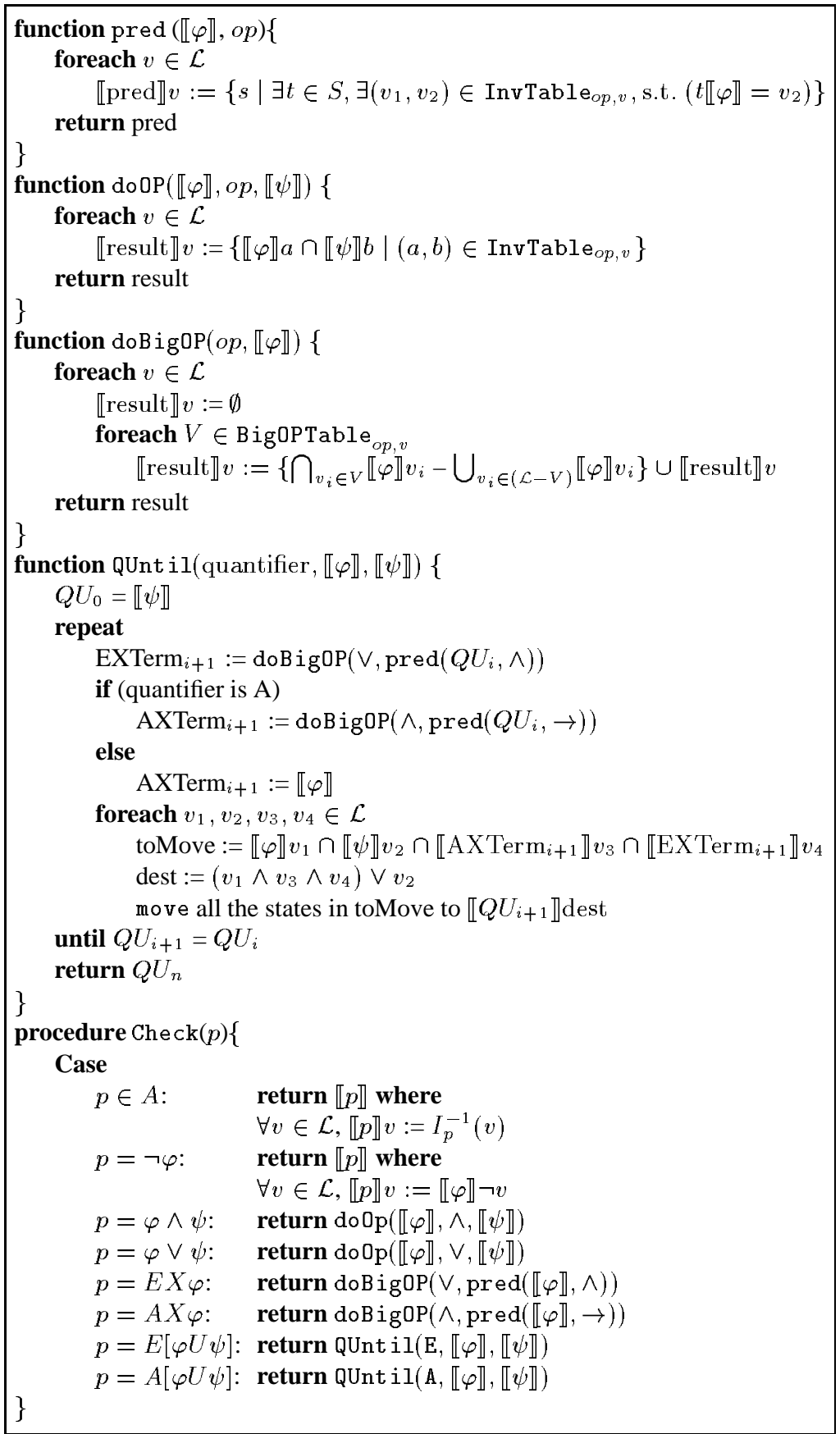

Fig. 6. Algorithm for $\chi_{\mathrm{chek}}$ 
call. Then, for each state $s$, the algorithm determines where this state should be by computing dest $:=s \llbracket \psi \rrbracket \vee\left(s \llbracket \varphi \rrbracket \wedge s \llbracket \mathrm{AXTerm}_{i+1} \rrbracket \wedge s \llbracket \mathrm{EXTerm}_{i+1} \rrbracket\right)$. If dest value is different from the one $s$ had in $Q U_{i}$, then it has to be moved to the appropriate place in $Q U_{i+1}$. The algorithm proceeds until no further changes to $Q U_{i}$ can be made.

For example, suppose we are computing $E[\neg$ Below $U$ Heat $]$ for our model in Figure 1(c) under the ordering T $<\mathrm{M}<\mathrm{F}$. $Q U_{0}$ is initialized to ( $\{\mathrm{HEAT}\},\{\mathrm{AC}\},\left\{\mathrm{OFF}, \mathrm{IDLE}_{1}\right.$, $\left.\left.\mathrm{IDLE}_{2}\right\}\right)$. IDLE $E_{2}$ has HEAT among its successors, so IDLE 2 【EXTerm $1 \rrbracket$ is T. Thus,

$$
\mathrm{IDLE}_{2} \llbracket \text { Heat } \rrbracket \vee\left(\mathrm{IDLE}_{2} \llbracket \neg \mathrm{Below} \rrbracket \mathrm{IDLE}_{2} \llbracket \mathrm{EXTerm}_{1} \rrbracket\right)=\mathrm{F} \vee(\mathrm{T} \wedge \mathrm{T})=\mathrm{T}
$$

and so IDLE $E_{2}$ should move to T. Using a similar process, we decide that dest for IDLE $E_{1}$ in $Q U_{1}$ is $\mathrm{M}$, and that dest for $\mathrm{AC}$ and $\mathrm{OFF}$ in $Q U_{2}$ are $\mathrm{T}$ and $\mathrm{M}$, respectively. The next iteration does not change $Q U_{2}$, and thus the algorithm terminates returning ( $\left\{\mathrm{HEAT}, \mathrm{AC}, \mathrm{IDLE}_{2}\right\}$, $\left.\left\{\mathrm{OFF}, \mathrm{IDLE}_{1}\right\},\{\}\right)$.

\begin{tabular}{l|l|c|c|c} 
Property & $\chi$ CTL formulation & Heater Model & AC Model & Combined Model \\
\hline Prop. 1. & $A G E X$ IDLE $_{1}$ & $\mathrm{~F}$ & $\mathrm{~T}$ & $\mathrm{~F}$ \\
Prop. 2. & $A[\neg$ Heat $U$ Below $]$ & $\mathrm{T}$ & - & $\mathrm{T}$ \\
Prop. 3. & $A G A F \neg$ Running & $\mathrm{F}$ & $\mathrm{F}$ & $\mathrm{F}$ \\
Prop. 4. & $A G$ (Heat $\leftrightarrow$ A ir) & - & - & $\mathrm{F}$ \\
Prop. 5. & $A G$ (Above $\rightarrow \neg$ Heat) & - & - & $\mathrm{M}$
\end{tabular}

Table 1. Results of verifying properties of the thermostat system.

The properties of the thermostat system that we identified in Section 2 can be translated into $\chi \mathrm{CTL}$ as described in Table 1 . The table also lists the values of these properties in each of the models given in Figure 1. We use "-" to indicate that the result cannot be obtained from this model. For example, the two individual models disagree on the question of reachability of state $\operatorname{IDLE}_{1}$ from every state in the model, whereas the combined model concludes that it is $\mathrm{F}$.

\section{Correctness and Termination of $\chi$ chek}

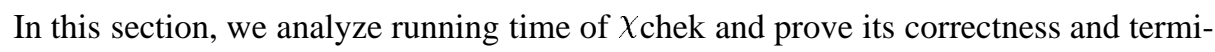
nation.

\subsection{Complexity}

Theorem 4 Procedure Check $(p)$ terminates on every $\chi C T L$ formula $p$.

Computation of Until takes the longest time. Each state can change its position in $\llbracket Q U_{i} \rrbracket$ at most $h$ times, where $h$ is the height of the lattice $(\mathcal{L}, \sqsubseteq)$. Thus, the maximum number of iterations of the loop in QUntil is $|S| \times h$. Each iteration takes the time to compute doBigOP on pred: $O\left(|\mathcal{L}| \times 2^{|\mathcal{L}|} \times|S|+|\mathcal{L}|^{2} \times|S|^{2}\right)$, plus the time to compute toMove and dest sets: $|\mathcal{L}|^{4} \times O(|S|)$. Therefore, the running time of Quntil is

$$
O\left(2^{|\mathcal{L}|} \times|S|^{2} \times|S| \times h\right)=O\left(2^{|\mathcal{L}|} \times|S|^{3}\right)
$$


and the running time of the entire model-checking algorithm on a property $p$ is

$$
O\left(2^{|\mathcal{L}|} \times|S|^{3} \times|p|\right)
$$

Note that in reality the running time is likely to be much smaller, because BigOPTable can be optimized and because set operations are BDD-based [9].

\subsection{Correctness}

In this section we prove correctness of $\chi$ chek by showing that it always returns exactly one answer (well-foundness) and that this answer is correct, i.e., it preserves the properties of $\chi$ CTL. We also show that multi-valued model-checking reduces to well-known boolean model-checking [24] if $(\mathcal{L}, \sqsubseteq)$ is the two-valued lattice representing classical logic.

We start by determining that procedure Check associates each state $s$ with exactly one logical value for each $\chi$ CTL property $p$.

Theorem 5 Procedure Check always returns a partition. Let $p$ be an arbitrary $\chi C T L$ formula. Then,

(a) $\forall s \in S, \exists v_{i} \in \mathcal{L}$, s.t. $s \in \llbracket \operatorname{Check}(p) \rrbracket v_{i} \quad$ (cover)

(b) $\forall s \in S, \exists v_{i}, v_{j} \in \mathcal{L}$, s.t.

$$
\left(s \in \llbracket \operatorname{Check}(p) \rrbracket v_{i} \wedge s \in \llbracket \operatorname{Check}(p) \rrbracket v_{j}\right) \rightarrow v_{i}=v_{j} \text { (disjointness) }
$$

Now we show that our algorithm preserves the expected properties of $\chi \mathrm{CTL}$ formulas given in Figure 4.

Theorem 6 Xchek preserves the negation of "next" property, i.e.

$$
\forall s \in S, s \in \llbracket \operatorname{Check}(A X \varphi) \rrbracket v \Leftrightarrow s \in \llbracket \operatorname{Check}(E X \neg \varphi) \rrbracket \neg v
$$

Theorem 7 Xchek preserves fixpoint properties of $A U$ and $E U$, i.e.

$$
\begin{gathered}
(1) \forall s \in S, s \llbracket \operatorname{Check}(A[\varphi U \psi]) \rrbracket=s \llbracket \operatorname{Check}(\psi) \rrbracket \vee \\
(s \llbracket \operatorname{Check}(\varphi) \rrbracket \wedge s \llbracket \operatorname{Check}(A X A[\varphi U \psi]) \rrbracket \wedge s \llbracket \operatorname{Check}(E X A[\varphi U \psi]) \rrbracket) \\
(2) \forall s \in S, s \llbracket \operatorname{Check}(E[\varphi U \psi]) \rrbracket= \\
s \llbracket \operatorname{Check}(\psi) \rrbracket \vee(s \llbracket \operatorname{Check}(\varphi) \rrbracket \wedge s \llbracket \operatorname{Check}(E X E[\varphi U \psi]) \rrbracket)
\end{gathered}
$$

( $\perp$ "until") follows easily from $A U$ and $E U$ fixpoints.

Our last correctness criterium is that the answers given by $\chi$ chek on $(2-\mathrm{Bool}, \sqsubseteq)$, a two-valued boolean lattice representing classical logic, are the same as given by a regular symbolic model-checker. We start by defining a "boolean symbolic model-checker" on Kripke structures, following [6] and changing some notation to make it closer to the one used in this paper. In particular, labeling functions used in boolean model-checking typically map a formula into a set of states where it is true, with the assumption that it is false in all other states. Thus, $\varphi$ maps into $\llbracket \varphi \rrbracket \top$ in our notation. The algorithm is given in Figure 7, with pre defined as follows:

$$
\operatorname{pre}(Q) \equiv\{s \mid t \in Q \wedge(s, t) \in R\}
$$

That is, $\operatorname{pre}(Q)$ computes all the states that can reach elements in $Q$ in one step. 


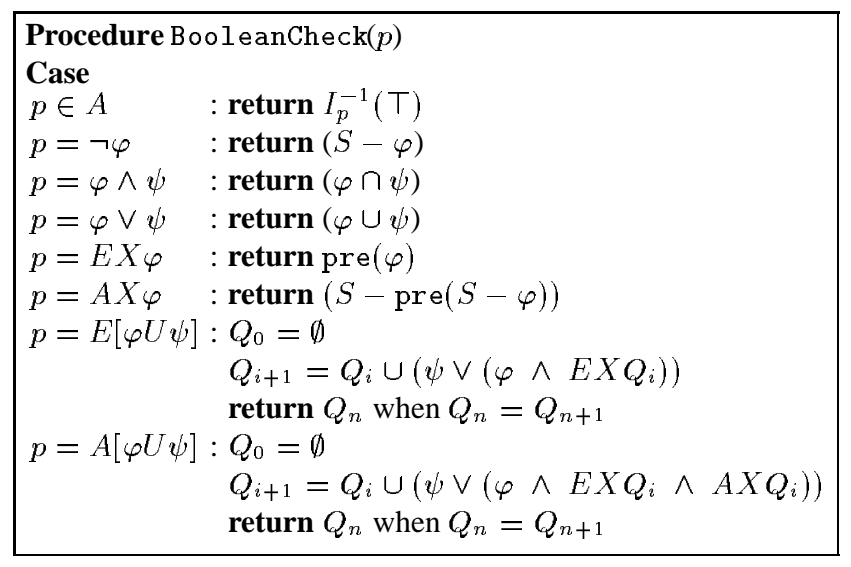

Fig. 7. Boolean Model-Checking Algorithm(adapted from [6]).

Theorem 8 Xchek, called on (2-Bool, $\sqsubseteq$ ), returns the same answers as a boolean modelchecker BooleanCheck, given in Figure 7. That is, for each $\chi \mathrm{CTL}$ property $p$ and each state $s \in S$,

$$
\begin{aligned}
& \text { (1) } s \in \llbracket \operatorname{Check}(p) \rrbracket \top \Rightarrow s \in \text { BooleanCheck }(p) \\
& \text { (2) } s \in \llbracket \operatorname{Check}(p) \rrbracket \perp \Rightarrow s \notin \text { BooleanCheck }(p)
\end{aligned}
$$

\section{Conclusion and Future Work}

Multi-valued logics are a useful tool for describing models that contain incomplete information or inconsistency. In this paper we presented an extension of classical CTL model-checking to reasoning about arbitrary quasi-boolean logics. We also described an implementation of a symbolic multi-valued model-checker $\chi$ chek and proved its termination and correctness.

We plan to extend the work presented here in a number of directions to ensure that $\chi$ chek can effectively reason about non-trivial systems. We will start by addressing some of the limitations of our $\chi$ Kripke structures. In particular, so far we have assumed that our variables are of the same type, with elements described by values of the lattice associated with that machine. We need to generalize this approach to variables of different types.

Further, in this work we have only addressed single-processor models. We believe that synchronous systems can be easily handled by our framework, and it is essential to extend our model-checking engine to reasoning about synchronous as well as asynchronous systems.

We are also in the process of defining and studying a number of optimizations for storage and retrieval of logic tables. These optimizations and the use of the $\chi \mathrm{DD}$ library do not change the worst-case running-time of $\chi$ chek, computed in Section 7. However, they significantly affect average-case running time. Once the implementation of the model-checker is complete, we intend to conduct a series of case studies to ensure that it scales up to reasoning about non-trivial systems.

Finally, we are interested in studying the properties of $\chi_{\text {chek in the overall frame- }}$ work of $\chi$ bel. This framework involves reasoning about multiple inconsistent descriptions of a system. We are interested in characterizing the relationship between the types 
of merge of individual descriptions and the interpretation of answers given by $\chi$ chek on the merged model.

\section{Acknowledgments}

We thank members of University of Toronto formal methods reading group, and in particular Ric Hehner, Albert Lai, Benet Devereux, Arie Gurfinkel, and Christopher ThompsonWalsh for numerous interesting and useful discussions and for careful readings of earlier drafts of this paper. We are also indebted to Albert and Benet for the proof of Theorem 2. Finally, we thank the anonymous referees for helping improve the presentation of this paper.

We gratefully acknowledge the financial support provided by NSERC and CITO.

\section{References}

1. A.R. Anderson and N.D. Belnap. Entailment. Vol. 1. Princeton University Press, 1975.

2. R.-J. Back and J. von Wright. Refinement Calculus: A Systematic Approach. Springer-Verlag, 1998.

3. N.D. Belnap. “A Useful Four-Valued Logic". In Dunn and Epstein, editors, Modern Uses of Multiple-Valued Logic, pages 30-56. Reidel, 1977.

4. L. Bolc and P. Borowik. Many-Valued Logics. Springer-Verlag, 1992.

5. R. E. Bryant. "Symbolic Boolean manipulation with ordered binary-decision diagrams". Computing Surveys, 24(3):293-318, September 1992.

6. T. Bultan, R. Gerber, and C. League. "Composite Model Checking: Verification with Type-Specific Symbolic Representations". ACM Transactions on Software Engineering and Methodology, 9(1):3-50, January 2000.

7. T. Bultan, R. Gerber, and W. Pugh. "Symbolic Model Checking of Infinite State Programs Using Presburger Arithmetic". In Proceedings of International Conference on ComputerAided Verification, Haifa, Israel, 1997.

8. M. Chechik. "On Interpreting Results of Model-Checking with Abstraction". CSRG Technical Report 417, University of Toronto, Department of Computer Science, September 2000.

9. M. Chechik, B. Devereux, and S. Easterbrook. "Implementing a Multi-Valued Symbolic Model-Checker". In Proceedings of TACAS'01, April 2001. to appear.

10. E.M. Clarke, E.A. Emerson, and A.P. Sistla. "Automatic Verification of Finite-State Concurrent Systems Using Temporal Logic Specifications". ACM Transactions on Programming Languages and Systems, 8(2):244-263, April 1986.

11. E.W. Dijkstra and C.S. Scholten. Predicate Calculus and Program Semantics. Springer, 1990.

12. D.L. Dill. "The Mur $\phi$ Verification System". In R. Alur and T.A. Henzinger, editors, Computer-Aided Verification Computer, volume 1102 of Lecture Notes in Computer Science, pages 390-393, New York, N.Y., 1996. Springer-Verlag.

13. J.M. Dunn. "A Comparative Study of Various Model-Theoretic Treatments of Negation: A History of Formal Negation". In Dov Gabbay and Heinrich Wansing, editors, What is Negation. Kluwer Academic Publishers, 1999.

14. S. Easterbrook and M. Chechik. "A Framework for Multi-Valued Reasoning over Inconsistent Viewpoints". In Proceedings of International Conference on Software Engineering (ICSE'01), May 2001. (to appear).

15. C. Ghezzi and B. A. Nuseibeh. "Introduction to the Special Issue on Managing Inconsistency in Software Development”. IEEE Transactions on Software Engineering, 24(11):906-1001, November 1998. 
16. S. Hazelhurst. Compositional Model Checking of Partially Ordered State Spaces. PhD thesis, Department of Computer Science, University of British Columbia, 1996.

17. S. Hazelhurst. "Generating and Model Checking a Hierarchy of Abstract Models". Technical Report TR-Wits-CS-1999-0, Department of Computer Science University of the Witwatersrand, Johannesburg, South Africa, March 1999.

18. E.C.R. Hehner. A Practical Theory of Programming. Texts and Monographs in Computer Science. Springer-Verlag, New York, 1993.

19. G.J. Holzmann. "The Model Checker SPIN". IEEE Transactions on Software Engineering, 23(5):279-295, May 1997.

20. A. Hunter. "Paraconsistent Logics". In D. Gabbay and Ph. Smets, editors, Handbook of Defeasible Reasoning and Uncertain Information, volume 2. Kluwer, 1998.

21. A. Hunter and B. Nuseibeh. "Managing Inconsistent Specifications: Reasoning, Analysis and Action". ACM Transactions on Software Engineering and Methodology, 7(4):335-367, October 1998.

22. M. Huth and M. Ryan. Logic in Computer Science: Modeling and Reasoning About Systems. Cambridge University Press, 2000.

23. S. C. Kleene. Introduction to Metamathematics. New York: Van Nostrand, 1952.

24. K.L. McMillan. Symbolic Model Checking. Kluwer Academic, 1993.

25. T.J. Menzies, S. Easterbrook, B. Nuseibeh, and S. Waugh. "An Empirical Investigation of Multiple Viewpoint Reasoning in Requirements Engineering”. In Proceedings of the Fourth International Symposium on Requirements Engineering (RE'99), Limerick, Ireland, June 711 1999. IEEE Computer Society Press.

26. G. Priest and K. Tanaka. "Paraconsistent Logic". In The Stanford Encyclopedia of Philosophy. Stanford University, 1996.

27. H. Rasiowa. An Algebraic Approach to Non-Classical Logics. Studies in Logic and the Foundations of Mathematics. Amsterdam: North-Holland, 1978.

\section{A Appendix}

In this appendix we give proofs for the theorems appearing in the main body of the paper. The proofs follow the calculational style of [11]. Section A.1 presents proofs of theorems of lattice theory; Section A.2 gives proofs of correctness of the definition of $\chi \mathrm{CTL}$ operators; Section A.3 lists properties of logic tables computed for $\chi_{\text {chek; finally, Sec- }}$ tion A.4 uses the properties given in Section A. 3 to prove correctness and termination of the implementation of $\chi$ chek.

\section{A.1 Lattice Theory}

Lattices have a number of properties that hold for them. We list several of them here, without proof.

$$
\begin{aligned}
& a \sqsubseteq b \Leftrightarrow \forall c, c \sqsubseteq a \Rightarrow c \sqsubseteq b \quad \text { (巨 introduction) } \\
& a \sqsubseteq b \Leftrightarrow \forall c, a \sqsubseteq c \Leftarrow b \sqsubseteq c \quad \text { (巨 introduction) } \\
& a \sqcap b \sqsubseteq b \text { and } a \sqcap b \sqsubseteq a \quad \text { ( } a \text { elimination) } \\
& a \sqsubseteq b \wedge a \sqsubseteq c \Rightarrow a \sqsubseteq b \sqcap c \quad \text { ( } \\
& a \sqsubseteq a \sqcup b \text { and } b \sqsubseteq a \sqcup b \quad \text { ( } \square \text { introduction) } \\
& a \sqsubseteq c \wedge b \sqsubseteq c \Rightarrow a \bar{\sqcup} b \sqsubseteq c \quad \text { (ப elimination) } \\
& a \sqsubseteq b \Leftrightarrow a \sqcup b=b \quad \text { (correspondence) } \\
& a \sqsubseteq b \Leftrightarrow a \sqcap b=a \quad \text { (correspondence) }
\end{aligned}
$$


The following are the properties of the product of two lattices $\left(\mathcal{L}_{1}, \sqsubseteq\right)$ and $\left(\mathcal{L}_{2}, \sqsubseteq\right)$ :

$$
\begin{aligned}
& \perp_{\mathcal{L}_{1} \times \mathcal{L}_{2}}=\left(\perp_{\left.\mathcal{L}_{1}, \perp_{\mathcal{L}_{2}}\right)}\right) \quad(\perp \text { of pairs }) \\
& T_{\mathcal{L}_{1} \times \mathcal{L}_{2}}=\left(T_{\mathcal{L}_{1}}, T_{\mathcal{L}_{2}}\right) \quad \text { ( } T \text { of pairs) } \\
& \neg(a, b)=(\neg a, \neg b) \quad \text { ( }) \text { of pairs) } \\
& (a, b) \sqcap\left(a^{\prime}, b^{\prime}\right)=\left(a \sqcap a^{\prime}, b \sqcap b^{\prime}\right) \quad(\sqcap \text { of pairs }) \\
& (a, b) \sqcup\left(a^{\prime}, b^{\prime}\right)=\left(a \sqcup a^{\prime}, b \sqcup b^{\prime}\right) \quad(\sqcup \text { of pairs) }
\end{aligned}
$$

Theorem 1. A product of two quasi-boolean lattices is quasi-boolean, that is,

(2) $\neg\left(\left(a_{1}, b_{1}\right) \sqcap\left(a_{2}, b_{2}\right)\right)=\left(\neg a_{1}, \neg b_{1}\right) \sqcup\left(\neg a_{2}, \neg b_{2}\right)$

(3) $\neg\left(\left(a_{1}, b_{1}\right) \sqcup\left(a_{2}, b_{2}\right)\right)=\left(\neg a_{1}, \neg b_{1}\right) \sqcap\left(\neg a_{2}, \neg b_{2}\right)$

(4) $\quad\left(a_{1}, b_{1}\right) \sqsubseteq\left(s_{2}, b_{2}\right) \Leftrightarrow \neg\left(a_{1}, b_{1}\right) \sqsupseteq \neg\left(a_{2}, b_{2}\right)$

Proof:

$$
\begin{aligned}
& \text { (1) } \neg \neg(a, b) \quad(2) \quad \neg\left(\left(a_{1}, b_{1}\right) \sqcap\left(a_{2}, b_{2}\right)\right) \\
& \Leftrightarrow \quad(\neg \text { of pairs) } \Leftrightarrow \quad \text { ( } \sqcap \text { of pairs) } \\
& \neg(\neg a, \neg b) \quad \neg\left(\left(a_{1} \sqcap a_{2}\right),\left(b_{1} \sqcap b_{2}\right)\right) \\
& \Leftrightarrow \quad(\neg \text { of pairs) } \\
& (\neg \neg a, \neg \neg b) \quad\left(\neg\left(a_{1} \sqcap a_{2}\right), \neg\left(b_{1} \sqcap b_{2}\right)\right) \\
& \Leftrightarrow \quad(\neg \text { involution }) \\
& (a, b) \\
& \begin{array}{ll}
\Leftrightarrow \quad & \text { (de Morgan) } \\
& \left(\neg a_{1} \sqcup \neg a_{2}, \neg b_{1} \sqcup \neg b_{2}\right) \\
\Leftrightarrow \quad & (\sqcup \text { of pairs) } \\
& \left(\neg a_{1}, \neg b_{1}\right) \sqcup\left(\neg a_{2}, \neg b_{2}\right)
\end{array}
\end{aligned}
$$

The proof of (3) is similar to that of (2).

Theorem 2. Let $(\mathcal{L}, \sqsubseteq)$ be a horizontally-symmetric lattice. Then the following hold for any two elements $a, b \in \mathcal{L}$ :

$$
\begin{aligned}
& H(a \sqcap b)=H(a) \sqcup H(b) \\
& H(a \sqcup b)=H(a) \sqcap H(b)
\end{aligned}
$$

\section{Proof:}

We will prove the first of these equations here, using the proof notation of [18]. The second one is a dual. We show

$$
\begin{aligned}
& \text { (1) } H(a \sqcap b) \sqsubseteq H(a) \sqcup H(b)(1) \\
& \text { (2) } H(a \sqcap b) \sqsupseteq H(a) \sqcup H(b)(2)
\end{aligned}
$$

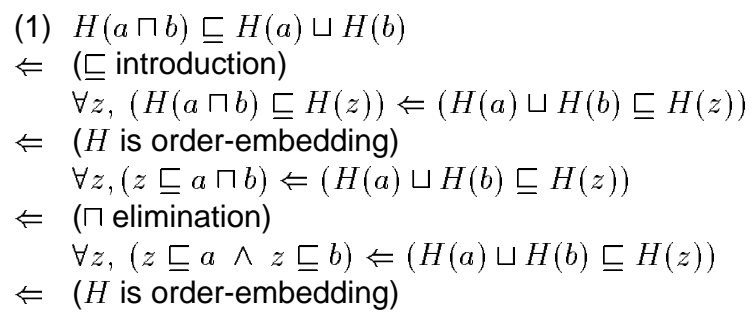




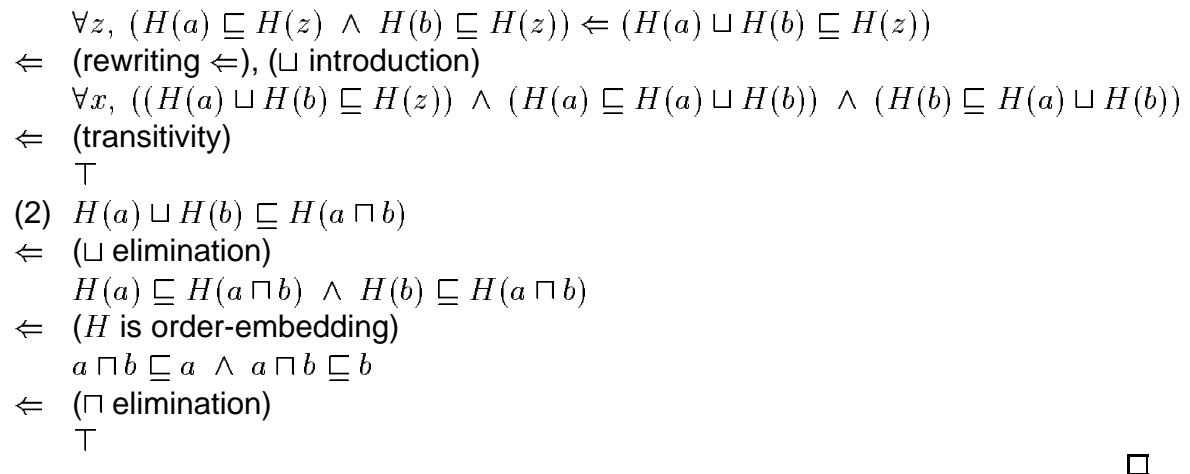

\section{A.2 $\chi$ CTL}

Theorem 3. Definitions of $s \llbracket E X \varphi \rrbracket$ and $s \llbracket A X \varphi \rrbracket$ preserve the negation of "next" property, i.e.

$$
\forall s \in S, \neg s \llbracket A X \varphi \rrbracket=s \llbracket E X \neg \varphi \rrbracket
$$

\section{Proof:}

Let $s \in S$ be an arbitrary state. Then,

$$
\neg s \llbracket A X \varphi \rrbracket
$$

$=($ definition of $A X)$ $\neg\left(\bigwedge_{t \in S}(R(s, t) \rightarrow t \llbracket \varphi \rrbracket)\right)$

$=$ (de Morgan), because $\neg$ is a quasi-boolean operator $\bigvee_{t \in S} \neg(R(s, t) \rightarrow t \llbracket \varphi \rrbracket)$

$=$ (definition of $\rightarrow$ ), (de Morgan) $\bigvee_{t \in S}(\neg \neg R(s, t) \wedge \neg t \llbracket \varphi \rrbracket)$

$=(\neg$ involution), because $\neg$ is a quasi-boolean operator $\bigvee_{t \in S}(R(s, t) \wedge \neg t \llbracket \varphi \rrbracket)$

$=$ (definition of $s \llbracket \neg \varphi \rrbracket)$ $\bigvee_{t \in S}(R(s, t) \wedge t \llbracket \neg \varphi \rrbracket)$

$=($ definition of $E X)$ $s \llbracket E X \neg \varphi \rrbracket$

\section{A.3 Table Library}

Here we give properties of inverse and BigOP tables defined in Section 6.1.

Lemma 1. The following are properties of inverse tables, with op $\in\{\wedge, \vee, \rightarrow\}$ :

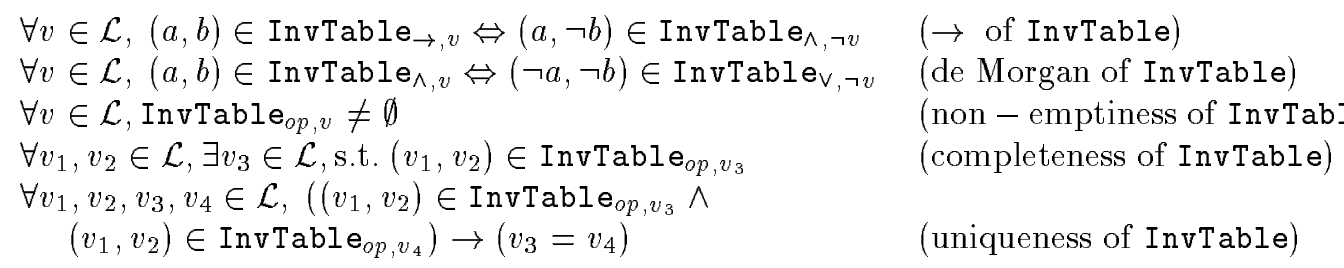




\section{Proof:}

From the definition of inverse tables, negation properties, the definition of $\rightarrow$ and lattice properties.

Lemma 2. The following are the properties of BigOP tables, with op $\in\{\wedge, \vee\}$ :

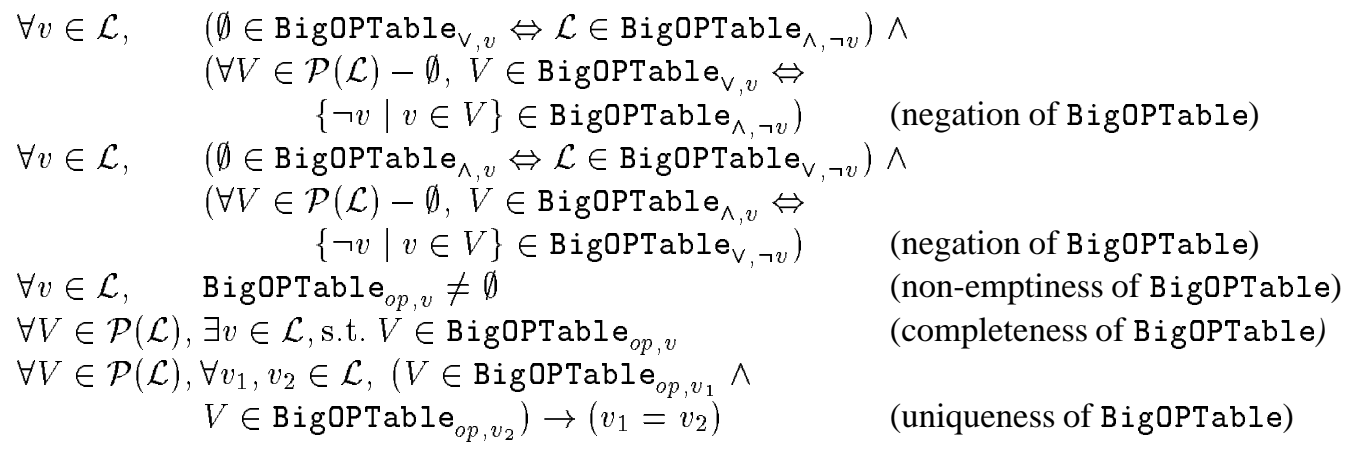

Proof:

By construction of BigOP tables and by the idempotency property of lattices.

Lemma 3. The following are the properties of predecessor relations:

$\forall \varphi, \forall s \in S, \exists v \in \mathcal{L}$, s.t. $s \in \llbracket \operatorname{pred}(\llbracket \varphi \rrbracket, o p) \rrbracket v$ (completeness of pred)

$\llbracket \operatorname{pred}(\llbracket \varphi \rrbracket, \wedge) \rrbracket v=\llbracket \operatorname{pred}(\llbracket \neg \varphi \rrbracket, \rightarrow) \rrbracket \neg v \quad$ (implication of pred)

\section{Proof:}

(completeness of pred)

Pick $\varphi$, pick a state $s$, pick a state $t$. Then, let $v_{1}=R(s, t)$ and $v_{2}=t \llbracket \varphi \rrbracket$. Further, let $v=v_{1}$ op $v_{2}$. Then, by the completeness of InvTable property, $s \in \llbracket \operatorname{pred}(\llbracket \varphi \rrbracket, o p) \rrbracket v$.

(implication of pred)

Let $s \in S$ be an arbitrary state. Then,

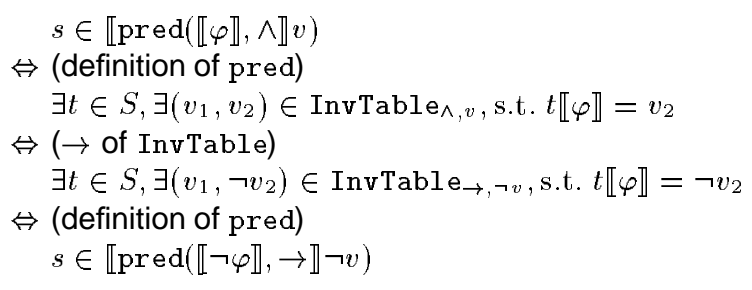

\section{A.4 Correctness and Termination}

Theorem 4. Procedure Check $(p)$ terminates on every $\chi C T L$ formula $p$.

\section{Proof:}

Proof is on the structure of property $p$. Obviously, for all operators except "until", Check $(p)$ terminates. We give proof for computing $A U$ here. To prove that the execution of QUnt il terminates, it suffices to show that $\forall s \in S, \forall i, s \llbracket Q U_{i} \rrbracket \sqsubseteq s \llbracket Q U_{i+1} \rrbracket$. Then, $Q U_{i}$ can change value at most $h$ times, where $h$ is the height of lattice $(\mathcal{L}, \sqsubseteq)$.

The proof goes by induction on $i$. Pick $s \in S$. Then, 


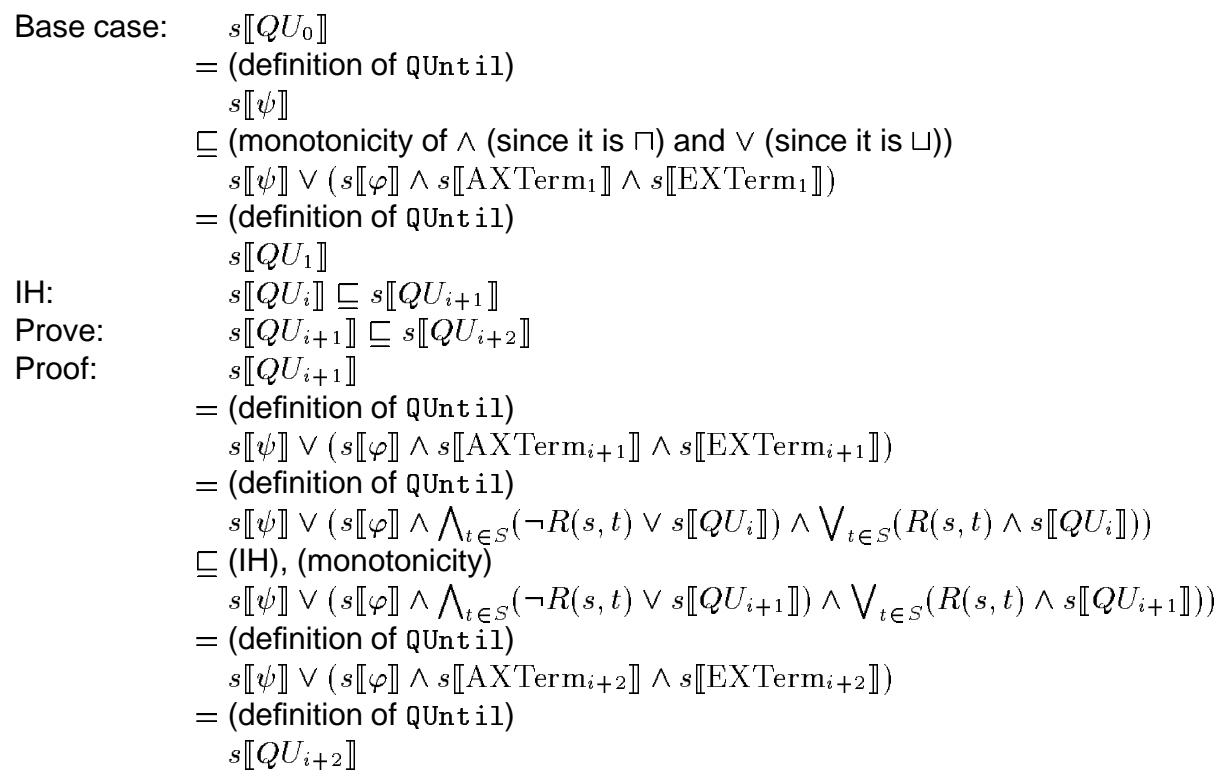

Theorem 5. Procedure Check always returns a partition. Let $p$ be an arbitrary $\chi C T L$ formula. Then,

(a) $\forall s \in S, \exists v_{i} \in \mathcal{L}$, s.t. $s \in \llbracket \operatorname{Check}(p) \rrbracket v_{i} \quad$ (cover)

(b) $\forall s \in S, \exists v_{i}, v_{j} \in \mathcal{L}$, s.t.

$\left(s \in \llbracket \operatorname{Check}(p) \rrbracket v_{i} \wedge s \in \llbracket \operatorname{Check}(p) \rrbracket v_{j}\right) \rightarrow v_{i}=v_{j}$ (disjointness)

\section{Proof:}

The proof is by induction on the length of $p$.

Base case: $p \in A$. Check $(p)$ uses $I$ which is guaranteed to return a partition by definition.

$\mathrm{IH}: \quad$ Assume $\operatorname{Check}(p)$ returns a partition when $|p| \leq n$.

Prove: $\quad \operatorname{Check}(p)$ returns a partition when $|p|=n+1$.

Proof:

$p=\neg \varphi \quad$ Then, $\llbracket \varphi \rrbracket$ is a partition by $\mathrm{IH}$, and negation is onto by $\neg$ involution.

$p=\varphi \wedge \psi \quad$ Pick state $s \in S$. Since $\llbracket \varphi \rrbracket$ and $\llbracket \psi \rrbracket$ are partitions, $\exists v_{1}, v_{2} \in \mathcal{L}$ s.t.

$s \in \llbracket \varphi \rrbracket v_{1}$ and $s \in \llbracket \psi \rrbracket v_{2}$.

(a) By completeness of InvTable, $\exists v_{3}$, s.t. $\left(v_{1}, v_{2}\right) \in \operatorname{InvTable}_{\wedge, v_{3}}$, so $s \in \llbracket p \rrbracket v_{3}$.

(b) By uniqueness of InvTable.

$p=\varphi \vee \psi \quad$ The proof is similar to the one above.

$p=E X \varphi . \quad$ Pick a state $s \in S$.

Create a set $V=\{v \mid s \in \llbracket \operatorname{pred}(\llbracket \varphi \rrbracket, \wedge) \rrbracket v\}$

(a) By completeness of BigOPTable, $\exists v_{i} \in \mathcal{L}$, s.t. $s \in \llbracket \operatorname{doBigOP}(\vee, \operatorname{pred}(\llbracket \varphi \rrbracket, \wedge)) \rrbracket v_{i}$.

(b) By uniqueness of BigOPTable, the above-found $v_{i}$ is unique.

$p=A X \varphi$. The proof is similar to the one above.

$p=E[\varphi U \psi]$ Partitionness is maintained as an invariant of QUntil: 
QUnt il starts of with a partition, and move preserves partition. $p=A[\varphi U \psi]$ Same as above.

Theorem 6. $\chi$ chek preserves the negation of "next" property, i.e.

$$
\forall s \in S, s \in \llbracket \operatorname{Check}(A X \varphi) \rrbracket v \Leftrightarrow s \in \llbracket \operatorname{Check}(E X \neg \varphi) \rrbracket \neg v
$$

\section{Proof:}

We prove

$$
\begin{aligned}
& \text { (1) } s \in \llbracket \operatorname{Check}(A X \varphi) \rrbracket v \quad \Rightarrow s \in \llbracket \operatorname{Check}(E X \neg \varphi) \rrbracket \neg v \\
& \text { (2) } s \in \llbracket \operatorname{Check}(E X \neg \varphi) \rrbracket \neg v \Rightarrow s \in \llbracket \operatorname{Check}(A X \varphi) \rrbracket v \\
& \text { (1) } s \in \llbracket \operatorname{Check}(A X \varphi) \rrbracket v \\
& \Rightarrow \text { (definition of Check) } \\
& s \in \llbracket \operatorname{doBigOP}(\wedge, \operatorname{pred}(\llbracket \varphi \rrbracket, \rightarrow)) \rrbracket v \\
& \Rightarrow \text { (definition of doBigOP) } \\
& \exists V \in \mathrm{BigOPTable}_{\wedge, v} \text {, s.t. }\left(s \in \bigcap_{v_{i} \in V} \llbracket \operatorname{pred}(\llbracket \varphi \rrbracket, \rightarrow) \rrbracket v_{i}\right) \wedge \\
& \Rightarrow \text { (negation of BigOPTable) } \\
& \left(s \notin \bigcup_{v_{i} \in(\mathcal{L}-V)} \llbracket \operatorname{pred}(\llbracket \varphi \rrbracket, \rightarrow) \rrbracket v_{i}\right) \\
& \exists V_{1} \in \mathrm{BigOPTable}_{\vee, \neg v} \text {, s.t. }\left(s \in \bigcap_{\neg v_{i} \in V_{1}} \llbracket \operatorname{pred}(\llbracket \varphi \rrbracket, \rightarrow) \rrbracket v_{i}\right) \wedge \\
& \Rightarrow \text { (implication of pred) } \\
& \left(s \notin \bigcup_{\neg v_{i} \in\left(\mathcal{L}-V_{1}\right)} \llbracket \operatorname{pred}(\llbracket \varphi \rrbracket, \rightarrow) \rrbracket v_{i}\right) \\
& \exists V_{1} \in \mathrm{BigOPTable}_{\vee, \neg v} \text {, s.t. }\left(s \in \bigcap_{\neg v_{i} \in V_{1}} \llbracket \operatorname{pred}(\llbracket \neg \varphi \rrbracket, \wedge) \rrbracket \neg v_{i}\right) \wedge \\
& \Rightarrow \text { (definition of doBigOP) } \\
& \left(s \notin \bigcup_{\neg v_{i} \in\left(\mathcal{L}-V_{1}\right)} \llbracket \operatorname{pred}(\llbracket \neg \varphi \rrbracket, \wedge) \rrbracket \neg v_{i}\right) \\
& s \in \llbracket \operatorname{doBigOP}(\vee, \operatorname{pred}(\llbracket \neg \varphi \rrbracket, \wedge)) \rrbracket \neg v \\
& \Rightarrow \text { (definition of Check) } \\
& s \in \llbracket \operatorname{Check}(E X \llbracket \neg \varphi \rrbracket) \rrbracket \neg v
\end{aligned}
$$

Proof of (2) is similar, and is based on implication of pred and negation of BigOPTable.

Theorem 7. $\chi$ chek preserves fixpoint properties of $A U$ and $E U$, i.e.

(1) $\forall s \in S, s \llbracket \operatorname{Check}(A[\varphi U \psi]) \rrbracket=s \llbracket \operatorname{Check}(\psi) \rrbracket \vee(s \llbracket \operatorname{Check}(\varphi) \rrbracket \wedge s \llbracket \operatorname{Check}(A X A[\varphi U \psi]) \rrbracket$ $\wedge s \llbracket \operatorname{Check}(E X A[\varphi U \psi]) \rrbracket)$

(2) $\forall s \in S, s \llbracket \operatorname{Check}(E[\varphi U \psi]) \rrbracket=s \llbracket \operatorname{Check}(\psi) \rrbracket \vee(s \llbracket \operatorname{Check}(\varphi) \rrbracket \wedge s \llbracket \operatorname{Check}(E X E[\varphi U \psi]) \rrbracket)$

\section{Proof:}

Pick a state $s$.

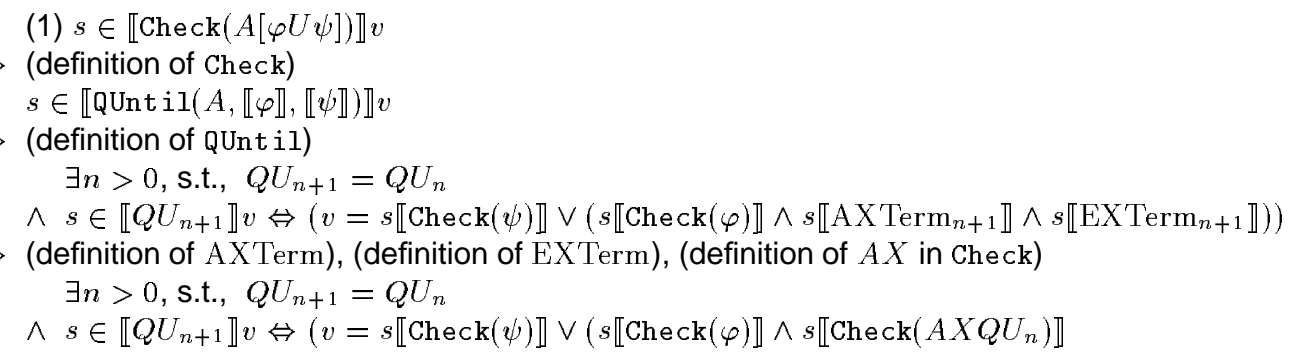




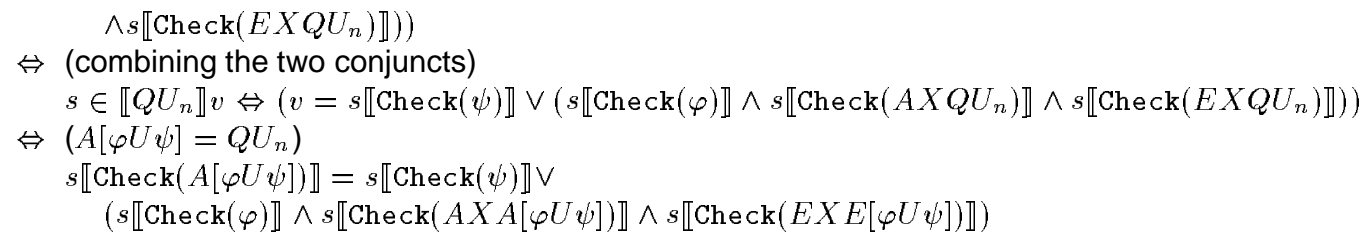

Proof of (2) is similar.

In our last theorem we want to prove that the result of calling $\chi$ chek with (2-Bool, $\sqsubseteq$ ), a lattice representing classical logic, is the same as the result of the boolean CTL model-checker.

We start by defining inverse and BigOP tables for a boolean lattice:

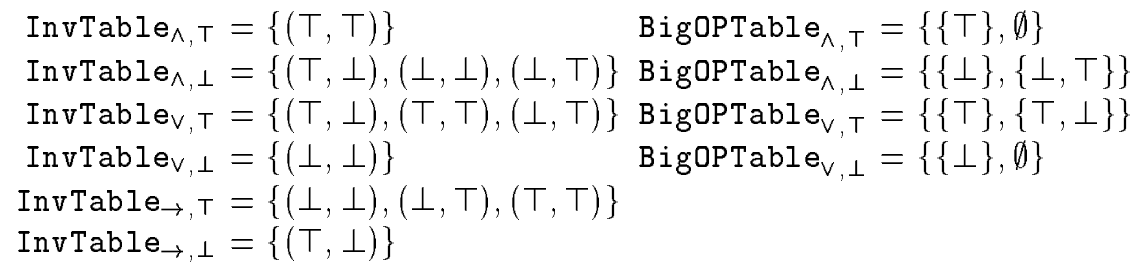

Lemma 4. The following relations holdfor each $s \in S$ when multi-valued model-checking is called on (2-Bool, $\sqsubseteq)$ :

$\forall s \in S, s \in \llbracket \operatorname{pred}(\llbracket \varphi \rrbracket, \wedge) \rrbracket \top \Rightarrow s \in \operatorname{pre}(\varphi) \quad$ (property of $\llbracket \operatorname{pred}(\llbracket \varphi \rrbracket, \wedge) \rrbracket \top)$

$\forall s \in S, s \in \llbracket \operatorname{pred}(\llbracket \varphi \rrbracket, \rightarrow) \rrbracket \top \Rightarrow s \in \neg \operatorname{pre}(\neg \varphi) \quad$ (property of $\llbracket \operatorname{pred}(\llbracket \varphi \rrbracket, \rightarrow) \rrbracket \top$ )

$\forall s \in S, s \in \llbracket \operatorname{pred}(\llbracket \varphi \rrbracket, \wedge) \rrbracket \perp \Rightarrow s \in \neg \operatorname{pre}(\varphi) \quad$ (property of $\llbracket \operatorname{pred}(\llbracket \varphi \rrbracket, \wedge) \rrbracket \perp)$

$\forall s \in S, s \in \llbracket \operatorname{pred}(\llbracket \varphi \rrbracket, \rightarrow) \rrbracket \perp \Rightarrow s \in \operatorname{pre}(\neg \varphi) \quad$ (property of $\llbracket \operatorname{pred}(\llbracket \varphi \rrbracket, \rightarrow) \rrbracket \perp$ )

\section{Proof:}

We prove properties of $\llbracket \operatorname{pred}(\llbracket \varphi \rrbracket, \wedge) \rrbracket \top$ and $\llbracket \operatorname{pred}(\llbracket \varphi \rrbracket, \rightarrow) \rrbracket \top$. The others follow from (implication of pred). For an arbitrary state $s \in S$,

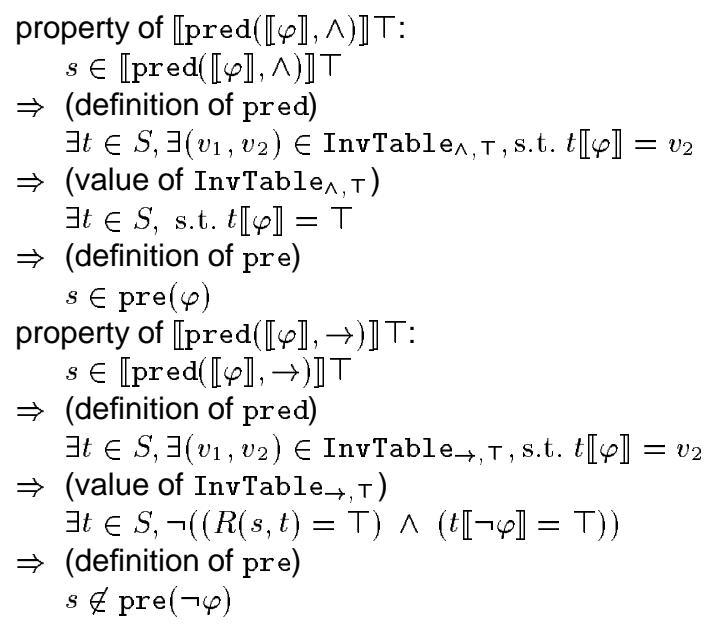




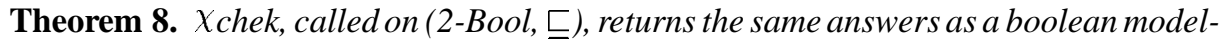
checker. That is, for each $\chi \mathrm{CTL}$ property $p$ and each state $s \in S$,

$$
\begin{aligned}
& \text { (1) } s \in \llbracket \operatorname{Check}(p) \rrbracket \top \Rightarrow s \in \operatorname{BooleanCheck}(p) \\
& \text { (2) } s \in \llbracket \operatorname{Check}(p) \rrbracket \perp \Rightarrow s \notin \operatorname{BooleanCheck}(p)
\end{aligned}
$$

\section{Proof:}

The proof is by induction on the structure of property $p$.

\section{Base Case:}

$p \in A: \operatorname{Check}(p)$ and BooleanCheck $(p)$ give the same answers by definition.

$\mathrm{IH}$ : $\quad$ Assume (1) and (2) hold for properties of length $\leq n$.

Prove: (1) and (2) hold for properties of length $n+1$.

Proof:

$$
\begin{aligned}
& p=\neg \varphi: \quad(1): s \in \llbracket \operatorname{Check}(p) \rrbracket \top \\
& \Rightarrow \text { (definition of Check) } \\
& s \in \llbracket \varphi \rrbracket \perp \\
& \Rightarrow \text { (definition of Check) } \\
& s \in \llbracket \operatorname{Check}(\varphi) \rrbracket \perp \\
& \Rightarrow \quad(\mathrm{IH}) \\
& s \notin \text { BooleanCheck }(\varphi) \\
& \Rightarrow \text { (definition of BooleanCheck) } \\
& s \in \text { BooleanCheck }(p) \\
& \text { Proof for (2) is similar } \\
& p=\varphi \wedge \psi: \quad(1): s \in \llbracket \operatorname{Check}(p) \rrbracket \top \\
& \Rightarrow \text { (definition of doOp) } \\
& s \in \llbracket \varphi \rrbracket a \wedge s \in \llbracket \psi \rrbracket b \wedge(a, b) \in{\text { InvTable } \mathrm{e}_{\wedge}, \mathrm{T}} \\
& \Rightarrow \text { (value of InvTable } e_{\wedge}, T \text { ) } \\
& s \in \llbracket \varphi \rrbracket \top \wedge s \in \llbracket \psi \rrbracket \top \\
& \Rightarrow \text { (changing notation) } \\
& s \in(\varphi \cap \psi) \\
& \Rightarrow \text { (definition of BooleanCheck) } \\
& s \in \text { BooleanCheck }(p) \\
& \text { Proof for (2) is similar. Because of the value of InvTable } e_{\wedge, \perp} \text {, } \\
& s \in \llbracket \varphi \rrbracket a \wedge s \in \llbracket \psi \rrbracket b \wedge(a, b) \in \text { InvTable }_{\wedge, \perp} \text { implies } \\
& \text { that } s \notin \llbracket \varphi \rrbracket \top \vee s \notin \llbracket \psi \rrbracket \top \text {. } \\
& p=\varphi \vee \psi \text { : Proofs are similar to the ones above and are based on values of } \\
& \text { InvTable } v, T \text { and InvTable }, \perp \text {. } \\
& p=E X \varphi: \quad(1): s \in \llbracket \operatorname{Check}(p) \rrbracket \top \\
& \Rightarrow \text { (definition of Check) } \\
& s \in \llbracket \operatorname{doB} \operatorname{igOP}(\vee, \operatorname{pred}(\llbracket \varphi \rrbracket, \wedge)) \rrbracket \top \\
& \Rightarrow \text { (definition of doBigOP), (value of BigOPTable } e_{\vee, T} \text { ) } \\
& \llbracket \text { result } \rrbracket \top=\emptyset \cup(\llbracket \operatorname{pred}(\varphi, \wedge) \rrbracket \top-\llbracket \operatorname{pred}(\varphi, \wedge) \rrbracket \perp) \\
& \cup(\llbracket \operatorname{pred}(\varphi, \wedge) \rrbracket \top \cap \llbracket \operatorname{pred}(\varphi, \wedge) \rrbracket \perp) \\
& \Rightarrow \quad\left(\operatorname{properties} \text { of } \llbracket \operatorname{pred}(\llbracket \varphi \rrbracket, \wedge) \rrbracket^{\top} \text { and } \llbracket \operatorname{pred}(\llbracket \varphi \rrbracket, \wedge) \rrbracket \perp\right) \\
& \llbracket \text { result } \rrbracket \top=(\operatorname{pre}(\varphi)-(\mathrm{S}-\operatorname{pre}(\varphi))) \cup(\operatorname{pre}(\varphi) \cap(\mathrm{S}-\operatorname{pre}(\varphi))) \\
& =\text { (set theory) } \\
& s \in \operatorname{pre}(\varphi)
\end{aligned}
$$




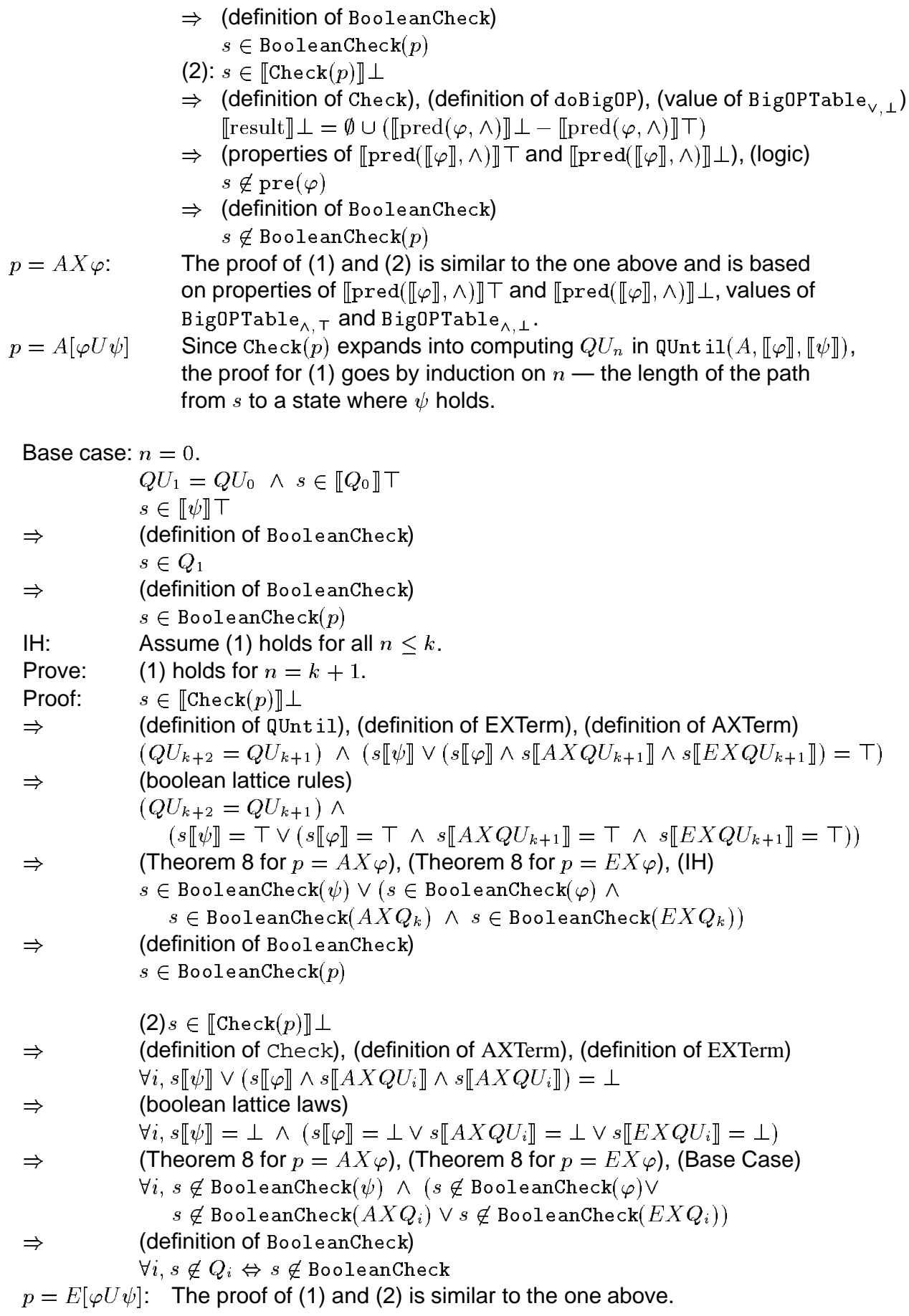

